Ann. Génét. Sél. anim., I980, 12 (1), 49-8o.

\title{
Diffusion du progrès génétique dans les populations avec générations imbriquées : quelques propriétés d'un modèle de prévision
}

\author{
J.M. ELSSEN \\ Station d'Amélioration Génétique des Animaux, \\ Centre de Recherches de Toulouse, I.N.R.A., \\ B.P. x2, Auzeville, 31320 Castanet Tolosan
}

\section{Résumé}

L'évolution de la valeur génétique moyenne d'une population sous l'effet de la sélection quand les générations se chevauchent a été décrite en 1974 par HILL et par EILSEN et MocQUOT à l'aide d'un modèle matriciel dont la formulation est proche des processus de MaRKov.

Dans cet article nous présentons de manière détaillée quelques propriétés intéressantes de ce modèle pour des populations hiérarchisées en sous-populations à vocations différentes : noyau de sélection, étage de multiplication et troupeaux de production.

En ce qui concerne les effets à long terme nous avons analysé le progrès annuel espéré dans chaque sous-population et les écarts entre les valeurs moyennes des animaux qui y naissent. Le cas d'une population à 2 étages est particulièrement détaillé et les expressions obtenues en I97I par BICHARD ("time lag") ont été généralisées.

D'un point de vue économique, ce sont les effets à court et moyen terme qui sont les plus importants. Le modèle proposé permet une formulation synthétique des recettes actualisées d'un schéma de sélection.

Cette formulation est simple mais implique des calculs importants dûs à une inversion de matrice. Pour une large utilisation dans les études de rentabilité, on donne ici une expression de ces recettes sans cet inconvénient. Il est même possible de calculer la valeur optimum de toute variable de đécision en dérivant cette expression.

Le développement des recherches sur la rentabilité des opérations de sélection a mis en évidence la nécessité d'une analyse fine de leurs répercussions sur la structure génétique des populations et en particulier sur l'évolution de la valeur génétique moyenne $\mathrm{du}$ ou des caractères à améliorer.

On a supposé tout d'abord que cette évolution était une fonction linéaire du temps (PouTous et VISSAC, I 962, I,INDHE, I968, HunT et a1., I974), dont la pente - ou progrès annuel - était aisément exprimable par une formule du type de celle de RENDEL et Robertson (r950). Cependant il est vite apparu qu'une telle évo- 
lution ne pouvait se produire que sous les hypothèses d'absence de migration et de générations séparées. Ainsi, dans le cas des populations dont les générations se chevauchent, SEARLE dès I96I, puis Hinks (1972), HILI (I97I), Mc ClinTOCK et CunNingham (I974) et BRASCAMp (I973) ont montré que la réponse à la sélection n'est pas linéaire du temps, tout au moins les premières années suivant la mise en place du programme de sélection. Par ailleurs, les populations hiérarchisées où tout l'effort de contrôle et d'amélioration est concentré sur une petite fraction (noyau) et où le progrès obtenu diffuse vers le reste des troupeaux (base) par migration, ont été étudiées par SMITH (I960), JACKSON et H.N. TURNER (1972) et surtout BICHARI (I97I) qui ont mis en évidence l'existence d'un décalage entre les valeurs moyennes du noyau et de la base.

Si ces phénomènes n'ont guère d'importance pour une prévision à long terme des conséquences de la sélection, il est par contre nécessaire d'en tenir compte quand on veut comparer la rentabilité de plusieurs plans de sélection. Ilans ce cas, en effet, le critère de comparaison sera généralement une fonction des recettes actualisées (Poutous et VISSAC I962, HrLL I97X), calculées comme la somme année par année des réponses à la sélection exprimées en unité monétaire. Or, par le biais de cette actualisation, on individualise les unes des autres les années (ou plus généralement les périodes élémentaires à partir desquelles on pourra décrire l'évolution de la valeur moyenne de la population comme un processus discret) et il convient alors de connaître aussi précisément que possible les réponses obtenues au cours de chaque période.

Un modèle, semble-t-il assez général, de prévision de cette évolution a été récemment proposé par HILL (I974) et ELSEN et MOCQUOT (I974). Nous voulons, dans cet article, en donner quelques propriétés. Après avoir défini les notations, nous rappellerons comment il permet de retrouver et généraliser les résultats à long terme. Puis nous montrerons comment obtenir une expression facilement calculable des recettes actualisées telles qu'elles ont été définies plus haut.

\section{I. - Le modèle - Rappels et notations}

Ce modèle est basé sur la décomposition de la population en groupes d'animaux de telle sorte que la valeur génétique additive moyenne du $i^{\text {eme }}$ groupe pour le caractère étudié au début de la période $t\left(\mathrm{X}_{i}(t)\right)$ puisse être déduite de l'ensemble des valeurs $\left(\mathrm{X}_{i}(t-\mathrm{I}), i=\mathrm{I}\right.$ à $\left.v\right)$ en $t-\mathrm{I}\left({ }^{*}\right)$. Par cette décomposition les animaux d'un même groupe seront en particulier de même sexe et âge (exprimé en nombre de périodes élémentaires). Une présentation détaillée des possibilités de décomposition est donnée par ELSEN et MocQvoT (I974). Alors, l'évolution de la population peut être décrite par une relation matricielle très simple : $\mathrm{X}(t)=$ $\mathrm{P}(t) \cdot \mathrm{X}(t-\mathrm{I})+\mathrm{V}(t)$ où $\mathrm{X}(t)$ est le vecteur des valeurs moyennes $\mathrm{X}_{i}(t), \mathrm{P}(t)$ une matrice de transition dont les éléments rendent compte du vieillissement et du renouvellement de la population et $\mathrm{V}(t)$ un vecteur d'écarts dus à la sélection. Il faut souligner avec HopkINs et JAmEs (I979) que les termes de la matrice $\mathrm{P}(t)$ dépendent aussi du schéma de sélection pratiqué et plus précisément de la sélection entre groupes d'animaux.

(*) Hul présente les éléments $\mathrm{X}_{i}(t)$ comme les proportions de gènes dans les génomes des animaux du $i e m e$ groupe en $t$ qui "dérivent " d'un groupe particulier en o. D'un point de vue opérationnel, compte tenu des mêmes hypothèses faites chez EISEN et MocQUOT, les résultats sont en tous points identiques. 
Cette relation de récurrence entre $\mathrm{X}(t-\mathrm{I})$ et $\mathrm{X}(t)$ résume en effet :

- le vieillissement de la population auquel correspondent des relations du type:

$$
\mathrm{X}_{t}(t)=\mathrm{X}_{j}(t-\mathrm{I})+\mathrm{V}_{i}(t)
$$

si $i$ est, par exemple, le groupe des mâles d'âge $k$ et $j$, d'âge $k-\mathrm{I}$

où :

$$
\mathrm{X}_{i}(t)=\sum_{j}\left(x_{i j}(t) \cdot \mathrm{X}_{j}(t)\right)+\mathrm{V}_{i}(t)
$$

si le $i^{\text {ìme }}$ groupe est formé dans les proportions $x_{i j}(t)$ par les animaux des groupes $j$,

- et le renouvellement de la population avec :

$$
\mathrm{X}_{i_{0}}(t)=\sum_{k}\left(a_{k}(t) \cdot \mathrm{X}_{k}(t-\mathrm{I})\right)+\sum_{l}\left(b_{l}(t) \cdot \mathrm{X}_{l}(t-\mathrm{I})\right)+\mathrm{V}_{i_{0}}(t)
$$

Si $i_{o}$ est le groupe des mâles d'âge $o, k$ les indices des groupes des pères de ces mâles et $l$ de leurs mères, $2 a_{k}(t)$ (resp. $2 b_{i}(t)$ ) la proportion, parmi les pères (resp. mères) des mâles d'âge $o$, de ceux qui appartiennent au groupe $k$ (resp. $l$ ), telle que $\sum_{k} a_{k}(t)=\frac{\mathrm{I}}{2} \quad\left(\right.$ resp. $\left.\sum_{l} b_{l}(t)=\frac{\mathrm{I}}{2}\right)$.

On aura de même $\mathrm{X}_{j_{0}}(t)=\sum_{k} c_{k}(t) \cdot \mathrm{X}_{k}(t-\mathrm{I})+\sum d_{l}(t) \cdot \mathrm{X}_{l}(t-1)+\mathrm{V}_{j_{0}}(t)$ pour le groupe $j_{o}$ des jeunes femelles.

Le vecteur des écarts $\mathrm{V}(t)$ est alors composé d'éléments qui représentent :

- dans les relations de type (I) la supériorité génétique des animaux du groupe $i$ par rapport à ceux du groupe $j$ due à une éventuelle sélection massale $\left(\mathrm{V}_{i}(t)\right.$ est nulle si aucun des mâles, par exemple, n'est éliminé volontairement entre les âges $k-\mathrm{I}$ et $k$ );

- dans les relations de type $(2) \mathrm{V}_{i}(t)=\sum_{j} x_{j}(t) \cdot \mathrm{V}_{i j}(t)$, où $\mathrm{V}_{i j}(t)$ est la supériorité génétique des animaux qui passent du groupe $j$ au groupe $i$ entre $t-\mathbf{I}$ et $t$;

- dans les relations de type (3) $\mathrm{V}_{i_{0}}(t)=\mathrm{V}_{i_{0} i_{0}}(t)+\sum_{k} a_{k}(t) \mathrm{V}_{i_{0} k}(t)+\sum_{l} b_{l}(t) \mathrm{V}_{i_{0} l}(t)$ où $\mathrm{V}_{i_{0} i_{0}}(t)$ est une composante chiffrant la sélection massale des jeunes animaux du groupe $i_{o}, \mathrm{~V}_{i_{0} k}(t)$ et $\mathrm{V}_{i_{0}}(t)$ sont des composantes correspondant à une sélection sur ascendance paternelle et maternelle.

A ce propos, il faut noter que contrairement à EL,SEN et MOCQUOT, HIL, n'introduit pas dans le vecteur $\mathrm{V}(t)$ cette composante due à la sélection sur ascendance, mais distingue par deux groupes de relations de récurrence complémentaires les voies pères-descendants et mères-descendants. En pratique ici aussi le choix est opérationnel et ne change pas les résultats.

En fait, les éléments du vecteur $\mathrm{X}(t)$ dont nous cherchons l'évolution dans le temps doivent être interprétés ici comme les valeurs génétiques additives moyennes des reproducteurs et non nécessairement de l'ensemble des animaux existants dans la population. $\mathrm{Si}$, en effet, on peut distinguer, dès la naissance et par les caractéristiques de leurs ascendants, les individus qui éventuellement se reproduiront de ceux qui ne le feront en aucun cas, il est possible que les pyramides des âges des parents des deux types d'animaux soient différentes. Fn d'autres termes, de même que nous avons défini plus haut les pondérations $a_{k}(t)$ et $b_{l}(t)$ pour les jeunes mâles et $c_{k}(t)$ et $d_{l}(t)$ pour les jeunes femelles, nous définirions 
maintenant des coefficients $p_{k}$ et $q_{l}$ pour l'ensemble des individus naissant au cours d'une période. Alors la valeur moyenne $\mathrm{Y}(t)$ de ces individus sera donnée par :

$$
\mathrm{Y}(t)=\sum_{k} p_{k} \cdot \mathrm{X}_{k}(t-\mathrm{I})+\sum_{l} q_{l} \cdot \mathrm{X}_{l}(t-\mathrm{I})
$$

que nous écrirons aussi $\mathrm{Y}(t)={ }^{t} r_{\pi} . \mathrm{X}(t--\mathrm{I}),{ }^{t r} \pi$ étant le vecteur ligne des $p_{k}$ et $q_{l}$.

Un exemple simple est donné dans le tableau I pour illustrer ce concept.

\section{TABLEAU I}

Exemple de population

Example of population

- On a alors besoin pour renouveler les reproducteurs de $60 \mathrm{mâles}$ et 400 femelles /période.

- Les candidats mâles à la reproduction sont choisis parmi les fils des pères de plus d'un an et des mères de plus de 2 ans.

- Les candidates femelles sont choisies uniquement sur ascendance maternelle : leurs mères doivent soit faire partie des $5^{\circ}$ p. I oo supérieures si elles ont I à 2 ans, soit avoir 2 à 3 ans.

- Alors, les coefficients seront :

$$
\begin{array}{lllll}
a_{1}=\mathrm{o} & a_{2}=\mathrm{I} / 2 & b_{1}=\mathrm{o} & b_{2}=\mathrm{o} & b_{3}=\mathrm{I} / 2 \\
c_{1}=\mathrm{I} / 6 & c_{2}=\mathrm{I} / 3 & d_{1}=\mathrm{o} & d_{2}=\mathrm{I} / 4 & d_{3}=\mathrm{I} / 4 \\
p_{1}=\mathrm{I} / 6 & p_{2}=\mathrm{I} / 3 & q_{1}=\mathrm{I} / 6 & q_{2}=2 / 9 & q_{\mathrm{3}}=\mathrm{I} / 9
\end{array}
$$

- Chaque période il naît donc 900 descendants dont 450 mâles et 450 femelles. Parmi ces 450 mâles, 60 se reproduiront au moins une fois et parmi ces $45^{\circ}$ femelles, 400 au moins 2 fois. Si $\mathrm{X}_{i}(t) i=\mathrm{I}$ à 5 sont les valeurs des reproducteurs en $t$ on aura d'une part :

d'autre part,

$$
\left[\begin{array}{ll}
\mathbf{X}_{1}(t) \\
\mathbf{X}_{2}(t) \\
\mathbf{X}_{3}(t) \\
\mathbf{X}_{4}(t) \\
\mathbf{X}_{5}(t)
\end{array}\right]=\left[\begin{array}{ccccc}
0 & \mathrm{I} / 2 & 0 & 0 & \mathrm{I} / 2 \\
\mathrm{I} & 0 & 0 & 0 & 0 \\
\mathrm{I} / 6 & \mathrm{I} / 3 & 0 & \mathrm{I} / 4 & \mathrm{I} / 4 \\
0 & 0 & \mathrm{I} & 0 & 0 \\
0 & 0 & 0 & \mathrm{I} & 0
\end{array}\right] \times\left[\begin{array}{l}
\mathrm{X}_{1}(t-\mathrm{I}) \\
\mathrm{X}_{2}(t-\mathrm{I}) \\
\mathrm{X}_{3}(t-\mathrm{I}) \\
\mathrm{X}_{4}(t-\mathrm{I}) \\
\mathrm{X}_{5}(t-\mathrm{I})
\end{array}\right]+\left[\begin{array}{l}
\mathrm{V}_{1} \\
\mathrm{~V}_{2} \\
\mathrm{~V}_{3} \\
\mathrm{~V}_{4} \\
\mathrm{~V}_{5}
\end{array}\right]
$$

$$
\mathrm{Y}(t)=(\mathrm{I} / 6, \mathrm{I} / 3, \mathrm{I} / 6,2 / 9, \mathrm{I} / 9) \times\left[\begin{array}{l}
\mathrm{X}_{1}(t-\mathrm{I}) \\
\mathrm{X}_{2}(t-\mathrm{I}) \\
\mathrm{X}_{3}(t-\mathrm{I}) \\
\mathrm{X}_{4}(t-\mathrm{I}) \\
\mathrm{X}_{5}(t-\mathrm{I})
\end{array}\right]
$$

Si on fait l'hypothèse que les caractéristiques démographiques de la population (mortalité, fertilité...) sont invariables dans le temps, que le schéma de sélection est répété sans changement d'une période à l'autre et que seules les espérances des 
distributions des valeurs génétiques et des prédicteurs de ces valeurs sont modifiées par la sélection, le modèle se simplifie considérablement et peut s'écrire :

$$
\mathrm{X}(t)=\mathrm{P} \cdot \mathrm{X}(t-\mathrm{I})+\mathrm{V}
$$

Ce sont les propriétés de ce modèle restreint qui nous intéressent ici. Par la suite, nous introduirons plusieurs types de matrices $\mathrm{P}$ correspondants à des structures de diffusion du progrès génétique différentes, où la population sera décomposée en sous-populations type noyaux, multiplicateurs... La notation $\mathrm{P}$ restera utilisée pour la matrice de transition entre tous les groupes de la population, mais on pourra éventuellement partitionner $\mathrm{P}$ en sous-matrices ${ }_{\alpha \beta} \mathrm{P}$ mettant en relation les sous-populations $\alpha$ et $\beta$.

On rencontrera alors deux types de sous-matrices : les sous-matrices ${ }_{\alpha \alpha} \mathrm{P}$ décrivant les phénomènes internes à la sous-population $\alpha$ et les sous-matrices ${ }_{\alpha \beta} \mathrm{P}$ décrivant les flux d'animaux entre $\alpha$ et $\beta$.

T1 Population fermée

T2 2 étages
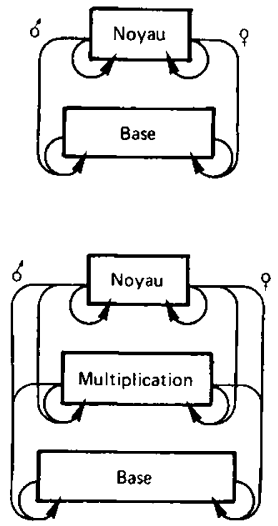

$$
P=\left(\begin{array}{cc}
11^{p} & 0 \\
21^{p} & 22^{p}
\end{array}\right)
$$

$$
P=\left(\begin{array}{ccc}
11^{p} & 0 & 0 \\
21^{p} & 22^{p} & 0 \\
31^{p} & 32^{p} & 33^{p}
\end{array}\right)
$$

T4 Croisement

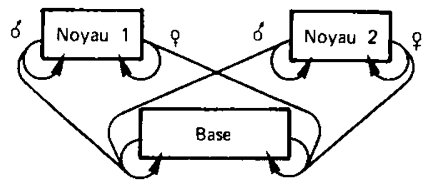

$$
P=\left(\begin{array}{ccc}
11^{p} & 0 & 0 \\
0 & 22^{p} & 0 \\
31^{p} & 32^{p} & 33^{p}
\end{array}\right)
$$

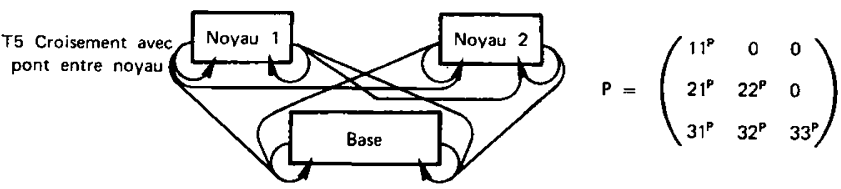

Les flèches indiquent les origines des animaux de renouvellement

FIG. I. - Populations étudiées Studied populations 
Les propriétés du modèle vont être démontrées pour cinq grands types de populations, schématisées figure $I$, auxquels correspondent cinq types de matrices de transition. Ce sont les populations fermées où tous les animaux sont contrôlés (TI), les populations hiérarchisées telles qu'elles ont été étudiées par BICHARD ( $197 \mathrm{I}$ ) avec ( $\mathrm{T}_{3}$ ) ou sans ( $\mathrm{T} 2$ ) étage de multiplication, les populations où on pratique le croisement ( $\left.\mathrm{T}_{4}\right)$ et un cas particulier montrant clairement une des propriétés du modèle $\left(\mathrm{T}_{5}\right)$.

Les matrices de transition correspondent à ces cinq situations sont des cas particuliers de la forme normale :

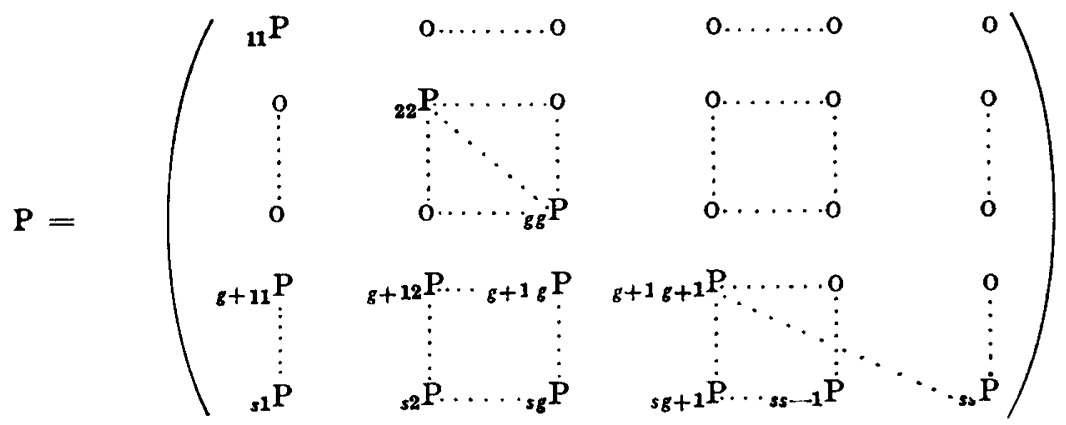

où ${ }_{\alpha \alpha} \mathrm{P}(\alpha<g)$ correspond au $\alpha^{i m e}$ noyau. ou base).

${ }_{\alpha \alpha} \mathrm{P}(\alpha>g)$, à la $\alpha^{\text {eme }}$ sous-population non fermée (étage de multiplication

${ }_{\alpha \beta} \mathrm{P}(\alpha>g, \beta<g)$ aux flux entre le noyau $\beta$ et la sous-population $\alpha$.

${ }_{\alpha \beta} \mathrm{P}(\alpha>g, g<\beta<\alpha)$, entre la sous-population $\beta$ et la sous-population $\alpha$.

Sous cette forme normale, les animaux de la sous-population ouverte $\alpha(\alpha>g)$ peuvent donc provenir des $g$ noyaux, de la sous-population $\alpha$ elle-même ou des $s-g-$ I autres sous-populations ouvertes.

Si la classification utilisée pour créer les groupes d'animaux tient compte de leur sous-population d'origine, ${ }_{\alpha \beta} \mathrm{P}$ et ${ }_{\alpha \alpha} \mathrm{P}(\alpha>g)$ se partitionnent en :

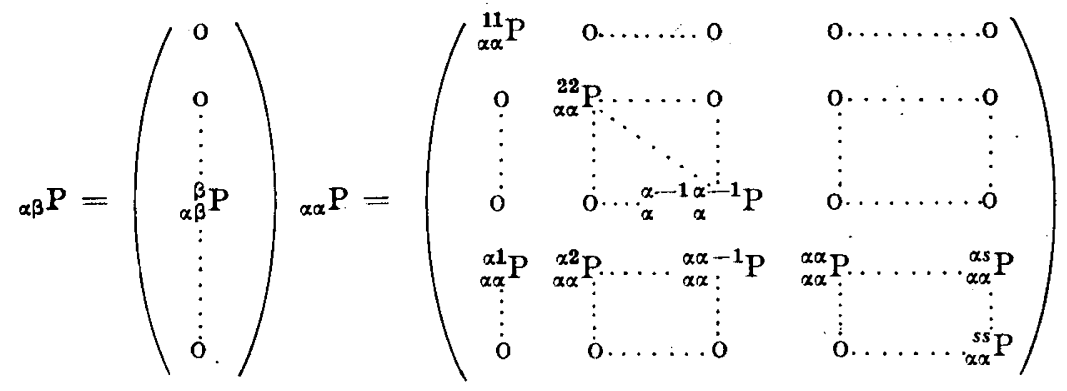

Sans perte de généralité, on pourra toujours supposer que toutes les sous-matrices ${ }_{\alpha \alpha} \mathrm{P}(\alpha \leqslant g),{ }_{\alpha \beta}^{\beta} \mathrm{P},{ }_{\alpha \alpha}^{\alpha \beta} \mathrm{P}$, et ${ }_{\alpha \alpha}^{\beta \beta} \mathrm{P}$ sont carrées et de même dimension $(n+m)^{2}, n$, étant le nombre de groupes de mâles et $m$, de femelles. En effet, les groupes correspondant à ces sous-matrices se distingueront uniquement par le sexe et l'âge (mâles de o à I an, de I à 2 ans, ... de $n$ - I à $n$ ans, femelles de o à I an ... de $m$ - I à $m$ ans). 
Pour simplifier les notations, nous noterons alors indifféremment ${ }_{\alpha \beta} Q$ toutes ces sous-matrices, $\alpha$ et $\beta$ variant maintenant de I à $g+s(s-g)$. Ainsi, pour les populations de type 2 :

$$
\mathrm{P}=\left(\begin{array}{cc}
{ }_{11} \mathrm{P} & 0 \\
{ }_{22} \mathrm{P} & { }_{22} \mathrm{P}
\end{array}\right)=\left(\begin{array}{ccc}
{ }_{11} \mathrm{P} & 0 & 0 \\
{ }_{21}^{1} \mathrm{P} & { }_{22}^{11} \mathrm{P} & 0 \\
0 & { }_{22} \mathrm{P} & { }_{22}^{22} \mathrm{P}
\end{array}\right)=\left(\begin{array}{ccc}
{ }_{11} \mathrm{Q} & 0 & 0 \\
{ }_{21} \mathrm{Q} & { }_{22} \mathrm{Q} & 0 \\
0 & { }_{32} \mathrm{Q} & { }_{33} \mathrm{Q}
\end{array}\right)
$$

et $\alpha \beta Q$ s'écrira de façon générale :

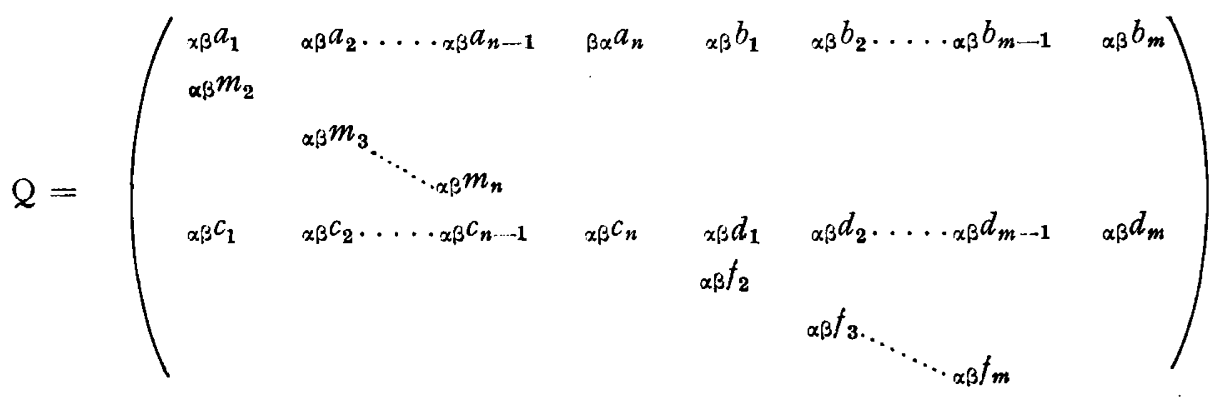

Par ailleurs, on appellera ${ }_{\alpha \beta} M$ la matrice tirée $\operatorname{des}{ }_{\alpha \beta} Q$ en annulant les ${ }_{\alpha \beta} m_{i}$ et les $\alpha \beta t_{j}$ :

$$
\mathrm{M}=\left(\begin{array}{cccccc}
{ }_{\alpha \beta} a_{1} & { }_{\alpha \beta} a_{2} \ldots \cdots \alpha_{\beta} a_{n} & { }_{\alpha \beta} b_{1} & { }_{\alpha \beta} b_{2} \cdots \cdots \alpha_{\beta} b_{m} \\
0 & 0 & 0 & 0 & 0 & 0 \\
\vdots & \vdots & \vdots & \vdots & \vdots & \vdots \\
{ }_{\alpha \beta} c_{1} & { }_{\alpha \beta} c_{2} \ldots{ }_{\alpha \beta} c_{n} & { }_{\alpha \beta} d_{1} & { }_{\alpha \beta} d_{2} \cdots \cdots \alpha_{\beta} d_{m} \\
0 & 0 & 0 & 0 & 0 & 0 \\
\vdots & \vdots & \vdots & \vdots & \vdots & \vdots \\
0 & 0 & 0 & 0 & 0 & 0
\end{array}\right)
$$

et ${ }_{\alpha \beta} \mathrm{H}$ la matrice ${ }_{\alpha \beta} \mathrm{H}=\left(\mathrm{I}-{ }_{\alpha \beta} \mathrm{Q}+{ }_{\alpha \beta} \mathrm{M}\right)^{-1}$

I étant la matrice unité, soit :
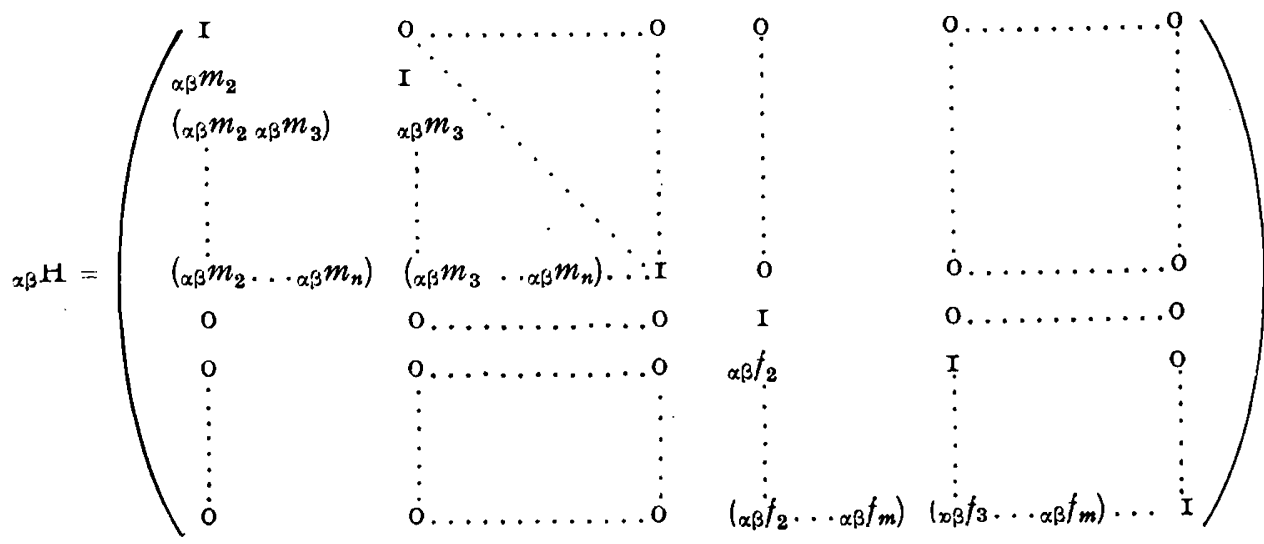
$\mathrm{Si}{ }_{\alpha \beta} \mathrm{Q}$ est une expression générale, il faut noter d'une part que tous les éléments des lignes I et $n+\mathrm{I}$ des matrices ${ }_{\alpha \alpha}^{\beta \beta} \mathrm{P}(\beta \neq \alpha)$ sont nuls et d'autre part, que seuls ces éléments ne sont pas nuls pour les matrices ${ }_{\alpha \alpha}^{\alpha \beta} \mathrm{P}(\beta \neq \alpha)$.

Plusieurs des propriétés du modèle font appel à l'expression directe de la matrice inverse $\left(\mathrm{I}-{ }_{\alpha \beta} Q\right)^{-1}$. La méthode pour obtenir cette expression est exposée en annexe $\mathrm{I}$ où les résultats suivants sont démontrés (pour la clarté, on a supprimé l'indicage $\alpha \beta$ : $a_{i}$ doit être $\operatorname{lu} \alpha \beta a_{i}$ ).

$$
\text { Si } \begin{aligned}
\mathrm{A}_{i} & =a_{i}+a_{i+i} m_{i+1}+\ldots+a_{n} m_{i+1} m_{i+2} \ldots m_{n} \\
\mathrm{~B}_{i} & =c_{i}+c_{i+1} m_{i+1}+\ldots+c_{n} m_{i+1} m_{i+2} \ldots m_{n} \\
\mathrm{C}_{i} & =b_{i}+b_{i+1} f_{i+1}+\ldots+b_{m} f_{i+1} \ldots f_{m} \\
\mathrm{D}_{i} & =d_{i}+d_{i+1} f_{i+1}+\ldots+d_{m} f_{i+1} \ldots f_{m}
\end{aligned}
$$

et $\quad \delta=\left(\mathrm{I}-\mathrm{A}_{1}\right)\left(\mathrm{I}-\mathrm{D}_{1}\right)-\mathrm{B}_{1} \cdot \mathrm{C}_{1}$

alors, $\left(\mathrm{I}-{ }_{\alpha \beta} \mathrm{Q}\right)^{-1}=\mathrm{J}+\mathrm{J} . \mathrm{F} . \mathrm{J}$ avec $\mathrm{J}=\left(\begin{array}{ll}\mathrm{JI} & 0 \\ 0 & \mathrm{~J} 2\end{array}\right)$
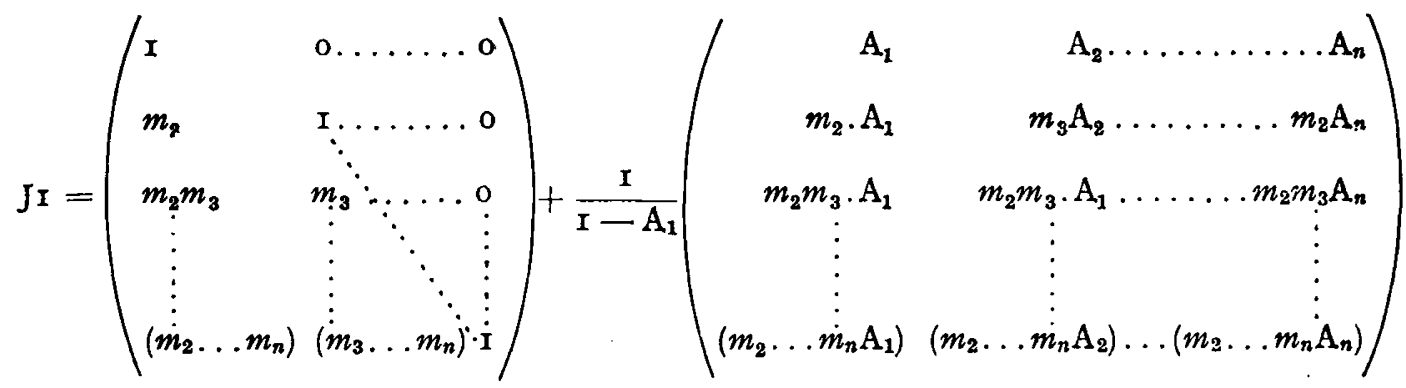

$\mathrm{J} 2$ semblable à $\mathrm{Jr}$ en remplaçant $m_{\iota}$ par $f_{i}$ et $\mathrm{A}_{i}$ par $\mathrm{D}_{i}$

et où

$\mathrm{F}=\frac{\mathrm{I}}{\delta} \times$
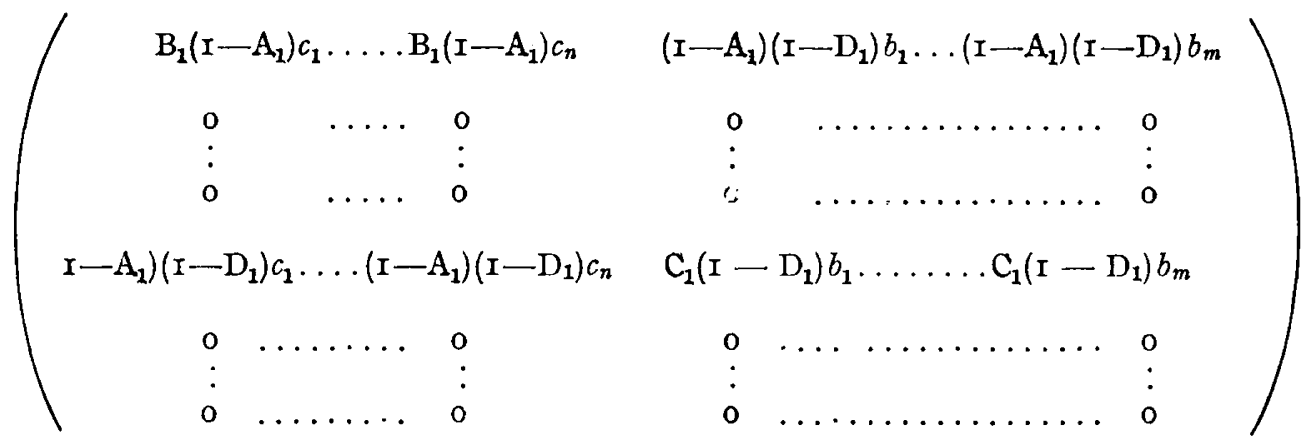

Comme la matrice $P$, les vecteurs $V$ et $\pi$ seront séparés en sous-vecteurs ${ }_{\alpha} V$ et $\alpha^{\pi}$ correspondant à chaque sous population. A l'intérieur d'un même sous-vecteur ${ }_{\alpha} \mathrm{V}$ on distinguera ${ }_{\alpha} \mathrm{V}^{\prime}$ pour les mâles de ${ }_{\alpha} \mathrm{V}^{\prime \prime}$ pour les femelles.

Par la suite, on notera $\alpha_{\alpha \beta} a,{ }_{\alpha \beta} b,{ }_{\alpha \beta} c,{ }_{\alpha \beta} d,{ }_{\alpha \beta} \mathrm{A},{ }_{\alpha \beta} \mathrm{B},{ }_{\alpha \beta} \mathrm{C}$, et ${ }_{\alpha \beta} \mathrm{D}$ les vecteurs d'éléments ${ }_{\alpha \beta} a_{i},{ }_{\alpha \beta} b_{i},{ }_{\alpha \beta} c_{i},{ }_{\alpha \beta} d_{i},{ }_{\alpha} \mathrm{A}_{i},{ }_{\alpha \beta} \mathrm{B}_{i},{ }_{\alpha \beta} \mathrm{C}_{i},{ }_{\alpha \beta} \mathrm{D}_{i}$; et $\mathcal{1}_{k}$ sera le vecteur colonne 
de dimension I $x k$ et dont les $k$ éléments sont égaux à I (si la dimension $k$ n'est pas spécifiée sera un vecteur à $n+m$ éléments). Par ailleurs $\alpha a$ (et de même ${ }_{\alpha} b,{ }_{\alpha} c$ et $\left.{ }_{\alpha} d\right)$ sera le vecteur $\sum_{\beta} \alpha_{\beta} a,{ }_{\alpha} \mathrm{A}$ et ${ }_{\alpha} \mathrm{A}_{i}$ étant
définis en conséquence. On notera également :

$$
\begin{aligned}
& { }_{\alpha \beta} \mathrm{G}_{\sigma^{\lambda}}{ }^{\alpha}=2 \cdot{ }_{\alpha}^{t r} \mathrm{~A} \cdot{ }_{\beta} \mathrm{V}^{\prime} \\
& { }_{\alpha \beta} \mathrm{G} \text { 웅 }=2 \cdot{ }_{\alpha}^{\text {tr }} \mathrm{B} .{ }_{\beta} \mathrm{V}^{\prime \prime} \\
& { }_{\alpha \beta} \mathrm{G}^{1}+\frac{1}{2}=2 \cdot{ }_{\alpha}^{t r} \mathrm{C}_{\beta} \mathrm{V}^{\prime} \\
& { }_{\alpha \beta} \mathrm{G} \text { 우 }=2 \cdot{ }_{\alpha}^{t r} \mathrm{D} \cdot{ }_{\beta} \mathrm{V}^{\prime \prime}
\end{aligned}
$$

et

$$
\begin{aligned}
& { }_{\alpha \beta} \mathrm{L}^{*} \sigma^{*}=2 \cdot{ }_{\alpha}^{t r} \mathrm{~A} . \quad 1_{n} \\
& { }_{\alpha \beta} \mathrm{L} \mathrm{L}_{\text {워 }}=2 \cdot{ }_{\alpha}^{t r} \mathrm{~B} . \quad 1_{m} \\
& { }_{\alpha \beta} \mathrm{L}_{\sigma}{ }^{\circ}+=2 \cdot{ }_{\alpha}^{t r} \mathrm{C} . \quad 1_{n} \\
& { }_{\alpha \beta} \mathrm{L} \text { 우우 }=2{ }_{\alpha}{ }_{\alpha} \mathrm{D} . \quad 1_{m}
\end{aligned}
$$

Dans le cas particulier où les voies pères fils et pères filles sont identiques $\left({ }_{\alpha} a={ }_{\alpha} b\right)$ on écrira :

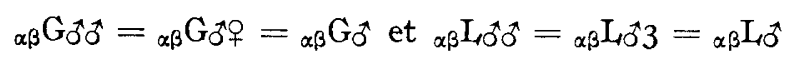

de même pour les voies mères fils et mères filles $\left({ }_{\alpha} c={ }_{\alpha} d\right)$

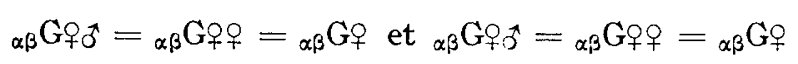

Enfin, 1'indicage $\alpha \beta$ sera omis pour les populations de type I ne comprenant $q u$ 'une sous-population.

\section{II. - Résultats à long terme}

\section{I. - Généralités}

La matrice $P$ est dans tous les cas stochastique et donc telle que sa ou ses plus grandes valeurs propres ont pour module I, et que le vecteur colonne I est vecteur propre de $P$.

Nous supposerons par la suite que les matrices ${ }_{\alpha \alpha} Q(\alpha<g)$ sont primitives, c'est-à-dire irréductibles et avec une seule valeur propre de module I. Cette hypothèse est discutée dans l'annexe 2 : sans être toujours réalisée, elle n'exclut qu'un petit nombre de cas assez particuliers.

Alors, la chaîne de MARKov correspondant à $P$ est régulière et $P$ peut s'écrire comme la somme $\mathrm{P}=\mathrm{R}+\mathrm{T}$ où $\mathrm{R}=\mathrm{P}^{\infty}$ (Gantmacher, I966). Comme $\mathrm{P} \mathrm{P}^{\infty}=$ $\mathrm{P}^{\infty} \mathrm{P}=\mathrm{P}^{\infty}, \mathrm{RT}=\mathrm{TR}=0$ et $\mathrm{P}^{t}=\mathrm{R}+\mathrm{T}^{t}$. Les lignes et les colonnes de $\mathrm{R}$ sont des vecteurs propres de $P$ pour la valeur propre $I$. Ces caractéristiques de la matrice $P$ permettent de donner deux propriétés essentielles du modèle. En effet, on a :

$$
\mathrm{X}(t+\mathrm{I})=\mathrm{P}^{t+1} \mathrm{X}(\mathrm{o})+\left(\mathrm{P}^{t}+\mathrm{P}^{t-1}+\ldots+\mathrm{I}\right) \mathrm{V}
$$

soit

$$
\mathrm{X}(t+\mathrm{I})=\mathrm{RX}(\mathrm{o})+t \mathrm{RV}+(\mathrm{I}-\mathrm{T})^{-1} \mathrm{~V}+\mathrm{T}^{t+1}\left(\mathrm{X}(\mathrm{o})-(\mathrm{I}-\mathrm{T})^{-3} \mathrm{~V}\right)
$$


L'effort cumulé (portant sur les $t$ premières années de sélection )est donc tel que : $\mathrm{X}(t+\mathrm{I}) \rightarrow \mathrm{RX}(\mathrm{o})+t \mathrm{RV}+(\mathrm{I}-\mathrm{T})^{-1} \mathrm{~V}$ quand $t \rightarrow \infty$ et comprend donc, outre $R X(o)$ correspondant au niveau initial, un terme d'écart $(I-T)^{-1} V$.

On peut par ailleurs isoler l'effet de la sélection pratiquée au cours de la seule année $t_{0}$ sur le niveau génétique moyen de l'année $t_{1}\left(t_{1}>t_{0}\right)$.

$$
\mathrm{S}\left(t_{o}, t_{1}\right)=\mathrm{P}^{t_{1}-t_{0}} . \mathrm{V}
$$

A long terme $\mathrm{S}\left(t_{0}, \infty\right)=\mathrm{R} . \mathrm{V}$ est indépendant de l'année considérée $t_{0}$.

La connaissance de la matrice $R=P^{\infty}$ est donc essentielle à la compréhension des effets de la sélection. Si on revient à la forme normale que nous résumerons maintenant par :

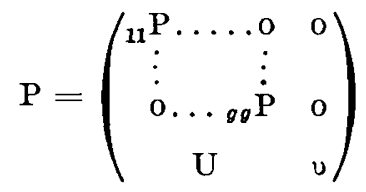

on sait (Cox et MILLER, I965) que R peut s'écrire :

où

$$
\mathrm{R}=\left(\begin{array}{cccc}
{ }_{11} \mathrm{P}^{\infty} & \ldots & 0 & 0 \\
\vdots & & \vdots & \vdots \\
0 & \ldots g g & \mathrm{P}^{\infty} & 0 \\
& \mathrm{U}_{\infty} & & 0
\end{array}\right)
$$

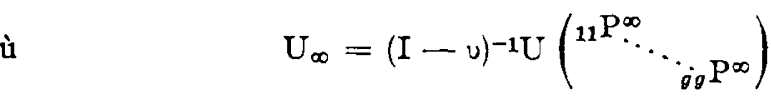

\section{2. - Effet d'une année de sélection}

A long terme, l'effet d'une année de sélection est donné par le produit RV. Selon le type de population, la matrice $\mathrm{R}$ prend des formes différentes.

\section{a) Population de type $\mathrm{I}$}

Dans ce cas, la matrice $P$ est primitive, donc n'a qu'une valeur propre de module $\mathrm{I}$ et le seul vecteur propre colonne de $\mathrm{P}$ correspondant à cette valeur propre est 1. R s'écrit alors ${ }^{t r} 1 . \phi^{\prime}\left({ }^{t r} 1\right.$ pour transposé de 1) où $\phi^{\prime}$ est vecteur propre ligne de P. HILL (I974) a montré que le vecteur $\phi=(\phi i)$ tel que :

$$
\begin{aligned}
& \phi i=\sum_{j=i}^{n}(a j+c j)=\mathrm{A}_{i}+\mathrm{C}_{i} \text { pour } i \leqslant n \\
& \phi i=\sum_{j=n-i}^{n+m}(b j+d j)=\mathrm{B}_{i}+\mathrm{D}_{i} \text { pour } i>n
\end{aligned}
$$

est vecteur propre de $P$.

Si on s'impose $\sum_{i=1}^{n+m} \phi^{\prime} i=\mathrm{I}$ ( $\mathrm{R}$ est stochastique), R s'écrit $\frac{1{ }^{t r} \phi}{{ }^{t r} 1 . \phi}$ et $\mathrm{RV}=1 \cdot \frac{{ }^{t r} \phi . \mathrm{V}}{{ }^{t r} 1 . \phi}$ 
HILL (I974), comme ELSEN et Mocquot (I974) par une voie différente, retrouve que $S_{\infty}=R V$ est un vecteur dont tous les éléments sont égaux au progrès génétique annuel $\mathrm{G}$ tel qu'il est défini par RENDEL et ROBER'TSON (I950) : $\mathrm{S}_{\infty}=1 . \mathrm{G}$.

b) Population de type 2

On a : $\mathrm{R}=\left(\begin{array}{ll}{ }_{11} \mathrm{P}^{\infty} & 0 \\ { }_{21} \mathrm{P}_{\infty} & 0\end{array}\right)$ avec ${ }_{11} \mathrm{P}^{\infty}=\frac{1 \cdot{ }_{11}^{t r} \phi}{{ }^{t r} 1_{11} \phi}{ }_{11} \phi$ étant défini comme $\phi$ et ${ }_{21} \mathrm{P}_{\infty}=\left(\mathrm{I}-{ }_{22} \mathrm{P}\right)^{-1}{ }_{21} \mathrm{P} \cdot{ }_{11} \mathrm{P}^{\infty}$

mais ${ }_{21} \mathrm{P} \cdot 1+{ }_{22} \mathrm{P} \cdot 1=1$. Donc $\left(\mathrm{I}-{ }_{22} \mathrm{P}\right)^{-1} \cdot{ }_{21} \mathrm{P} \cdot 1=1$. Soit ${ }_{21} \mathrm{P}_{\infty}={ }_{11} \mathrm{P}^{\infty}$.

Donc, ici aussi $S_{\infty}$ est un vecteur dont tous les éléments sont égaux à :

$$
{ }_{11} \mathrm{G}=\frac{{ }_{11}^{t r} \phi \cdot{ }_{1} \mathrm{~V}}{{ }^{t r} 1_{\cdot 11} \phi} \quad\left(\text { en notant } \mathrm{V}=\left(\begin{array}{l}
1 \\
\mathrm{~V} \\
\mathrm{~V}
\end{array}\right)\right)
$$

c) Population de type 3

on a encore : $\quad \mathrm{R}=\left(\begin{array}{lll}{ }_{11} \mathrm{P}^{\infty} & 0 & 0 \\ { }_{21} \mathrm{P}_{\infty} & 0 & 0 \\ { }_{31} \mathrm{P}_{\infty} & 0 & 0\end{array}\right)$

avec : $\quad\left(\begin{array}{l}{ }_{21} \mathrm{P}_{\infty} \\ { }_{31} \mathrm{P}_{\infty}\end{array}\right)=\left(\begin{array}{cc}\mathrm{I}-{ }_{22} \mathrm{P} & \mathrm{O} \\ -{ }_{32} \mathrm{P} & \mathrm{I}-{ }_{33} \mathrm{P}\end{array}\right)^{-1} \times\left(\begin{array}{l}{ }_{21} \mathrm{P} \\ { }_{31} \mathrm{P}\end{array}\right) \times{ }_{11} \mathrm{P}^{\infty}$

soit : $\quad{ }_{12} \mathrm{P}_{\infty}=\left(\mathrm{I}-{ }_{22} \mathrm{P}\right)^{-1} \cdot{ }_{21} \mathrm{P} \cdot{ }_{11} \mathrm{P}^{\infty}={ }_{11} \mathrm{P}^{\infty}$

et

$$
\begin{aligned}
& { }_{31} \mathrm{P}_{\infty}=\left(\left(\mathrm{I}-{ }_{33} \mathrm{P}\right)^{-1}{ }_{32} \mathrm{P}\left(\mathrm{I}-{ }_{22} \mathrm{P}\right)^{-1}{ }_{21} \mathrm{P}+\left(\mathrm{I}-{ }_{33} \mathrm{P}\right)^{-1}{ }_{31} \mathrm{P}\right){ }_{11} \mathrm{P}^{\infty} \\
& { }_{31} \mathrm{P}_{\infty}=\left(\left(\mathrm{I}-{ }_{33} \mathrm{P}\right)^{-1}{ }_{32} \mathrm{P}+\left(\mathrm{I}-{ }_{33} \mathrm{P}\right)^{-1}{ }_{31} \mathrm{P}\right){ }_{11} \mathrm{P}^{\infty}
\end{aligned}
$$

or $\quad{ }_{31} \mathrm{P} .1+{ }_{32} \mathrm{P} .1+{ }_{33} \mathrm{P} .1=\mathrm{I} \quad$ donc $\quad\left(\mathrm{I}-{ }_{33} \mathrm{P}\right)^{-1}\left({ }_{31} \mathrm{P}+{ }_{32} \mathrm{P}\right) \mathbf{1}=1$

Ici encore, $S_{\infty}$ est un vecteur dont tous les éléments sont égaux à ${ }_{11} G=\frac{{ }_{11}^{t r} \phi \cdot{ }_{1} \mathrm{~V}}{{ }^{t} 1_{\cdot 11} \phi}$.

Il apparait donc pour ces populations de type 2 et 3 qu'à long terme, seule la sélection pratiquée dans le noyau a un effet durable et que l'ensemble de la population progresse à la même vitesse, quel que soit le nombre d'étages de démultiplication entre le noyau et la base, et quelle que soit la méthode de diffusion des reproducteurs du moment qu'il n'y a pas de remontée vers le noyau. On retrouve donc ici en les généralisant les résultats obtenus par BICHARD (I97I).

\section{d) Population de type 4}

La matrice $P$ n'est pas primitive car I est racine double de l'équation caractéristique, chacune de ces valeurs propres correspondant à un noyau de sélection. Dans ces conditions, les lignes de $\mathrm{R}$ ne sont pas toutes identiques mais :

$$
\mathrm{R}=\left(\begin{array}{ccc}
{ }_{11} \mathrm{P}^{\infty} & 0 & 0 \\
0 & { }_{22} \mathrm{P}^{\infty} & 0 \\
\left(\mathrm{I}-{ }_{33} \mathrm{P}\right)^{-1}{ }_{31} \mathrm{P}_{11} \mathrm{P}^{\infty} & \left(\mathrm{I}-{ }_{33} \mathrm{P}\right)^{-1}{ }_{32} \mathrm{P}_{22} \mathrm{P}^{\infty} & 0
\end{array}\right)
$$


et 1 'effet à long terme est donné par :

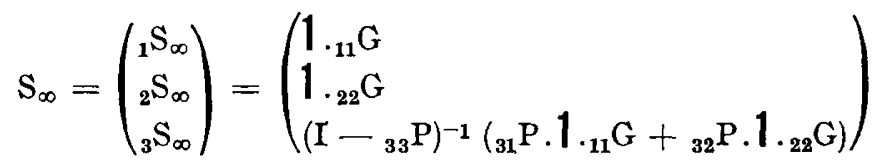

Le progrès espéré chez les animaux croisés est une combinaison linéaire des progrès réalisés dans les noyaux $\left({ }_{11} \mathrm{G}\right.$ et $\left.{ }_{22} \mathrm{G}\right)$, les coefficients de cette combinaison dépendant du type de diffusion pratiquée, caractérisée par ${ }_{31} \mathrm{P}$ et ${ }_{32} \mathrm{P}$.

Si maintenant on partitionne $P$ en sous-matrices élémentaires $\alpha \beta Q$, soit :

$$
P=\left(\begin{array}{rrrrr}
{ }_{11} Q & O & O & O & 0 \\
O & { }_{22} Q & O & O & 0 \\
{ }_{31} Q & O & { }_{33} Q & O & O \\
O & { }_{42} Q & O & { }_{44} Q & O \\
O & O & { }_{52} Q & { }_{53} Q & { }_{55} Q
\end{array}\right)
$$

on trouve que l'espérance du progrès annuel à long terme chez les croisés est :

$$
\begin{aligned}
{ }_{5} \mathrm{~S}_{\infty} & =\left(\mathrm{I}-{ }_{55} \mathrm{Q}\right)^{-1}\left[{ }_{53} \mathrm{Q} \cdot\left(\mathrm{I}-{ }_{33} \mathrm{Q}\right)^{-1} \cdot{ }_{31} \mathrm{Q} \cdot 1 \cdot{ }_{11} \mathrm{G}+{ }_{54} \mathrm{Q} \cdot\left(\mathrm{I}-{ }_{44} \mathrm{Q}\right)^{-1}{ }_{42} \mathrm{Q} \cdot 1 \cdot{ }_{22} \mathrm{G}\right] \\
& =\left(\mathrm{I}-{ }_{55} \mathrm{Q}\right)^{-1}\left[{ }_{53} \mathrm{Q} \cdot 1 \cdot{ }_{11} \mathrm{G}+{ }_{54} \mathrm{Q} \cdot 1 \cdot{ }_{22} \mathrm{G}\right]
\end{aligned}
$$

Le cas du croisement simple où les mâles proviennent du noyau I et les femelles du noyau 2 peut être symbolisé par :

$$
\sum_{i=1}^{n}{ }_{53} a_{i}=\sum_{i=1}^{n}{ }_{53} c_{i}=\sum_{j=1}^{m}{ }_{54} b_{j}=\sum_{j=1}^{m}{ }_{54} d_{j}=\mathrm{I} / 2
$$

et

$$
{ }_{55} m_{i}=\mathrm{I} \text { pour tout } i=2, n ;{ }_{55} f_{j}=\mathrm{I} \text { pour tout } j=2, m
$$

Avec ces hypothèses ${ }_{55} A_{1}={ }_{55} B_{1}={ }_{55} C_{1}={ }_{55} D_{1}=0$

et $\left(\mathrm{I}-{ }_{55} \mathrm{Q}\right)^{-1}={ }_{55} \mathrm{H} \quad\left[\begin{array}{c}{ }_{11} \mathrm{G} / 2 \\ 0 \\ 0 \\ { }_{11} \mathrm{G} / 2 \\ 0 \\ 0\end{array}\right]$ et ${ }_{54} \mathrm{Q} \cdot \mathrm{I}_{{ }_{22} \mathrm{G}}=\left[\begin{array}{c}{ }_{22} \mathrm{G} / 2 \\ 0 \\ 0 \\ { }_{22} \mathrm{G} / 2 \\ 0 \\ 0\end{array}\right]$

on voit que ${ }_{5} S_{\infty}=1 . \frac{\left({ }_{11} G+{ }_{22} G\right)}{2}$, comme on l'attend classiquement.

e) Population de type 5 $\left(^{*}\right)$

Il s'agit ici d'une extension du type 4 où une partie des reproducteurs du noyau 2 vient du noyau $I$ : ce serait par exemple le cas d'un plan de sélection où le

(*) Nous remercions J. W. James pour ses suggestions faites sur cette partie. 
noyau I serait sélectionné sur un groupe de caractère $A$ et le noyau 2 sur un autre groupe $B$ et où on envisagerait de récupérer dans le noyau 2 les animaux du noyau $\mathrm{I}$ ayant de bonnes performances en $B$.

L'examen des flux de reproducteurs (fig. I) montre que cette situation est en fait identique aux populations de type 3 . On peut donc en conclure que seule la sélection pratiquée dans le noyau I a une incidence à long terme, l'ensemble de la population progressant à la même vitesse ${ }_{11} \mathrm{G}$.

Si la corrélation entre les caractères $A$ et $B$ est négative, on peut donc avoir un résultat défavorable.

Notons que cette situation est celle des bovins laitiers où une partie des mâles est achetée dans les souches Holstein. Tant que cette importation durera, il ne sera pas possible d'orienter à long terme l'évolution des caractères et ceci, même si on sélectionne les reproducteurs de souche française utilisés dans ces schémas. Cette sélection peut modifier à court terme le niveau moyen des mâles pour les caractères dont l'intérêt est propre au pays, mais, à long terme, l'évolution sera parallèle à celle des souches Holstein.

\section{3. - Écarts à long terme}

Si au bout d'un temps suffisamment long l'ensemble des groupes progresse à vitesse constante (donné par le vecteur $S_{\infty}=R V$ ), il subsiste entre ces groupes un écart constant, donc relativement de moins en moins important, donné par le vecteur $(\mathrm{I}-\mathrm{T})^{-\mathbf{1}} \mathrm{V}=(\mathrm{I}-\mathrm{P}+\mathrm{R})^{-\mathbf{1}} \mathrm{V}$. C'est cet écart que BICHARD a étudié

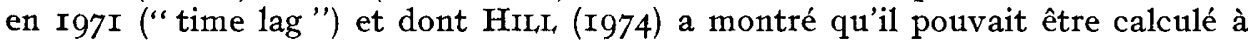
partir de la matrice $(\mathrm{I}-\mathrm{T})^{-1}$. I1 est possible, connaissant l'expression de cette inverse, de généraliser les résultats de BICHARD.

En pratique, ce n'est pas tant la valeur de $e=(\mathrm{I}-\mathrm{T})^{-\mathbf{1}} \mathrm{V}$ qui nous intéresse que les différences entre groupes qu'on pourra chiffrer par des produits du type de $\operatorname{tr\xi } . e$ où ${ }^{t r \xi}$ est soit un vecteur ligne ${ }^{t r \xi} \xi_{i j}$ dont tous les éléments sont nuls sauf le

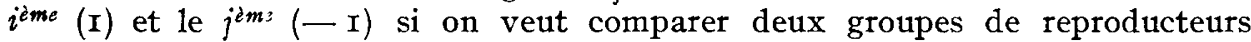
intra sous-population, soit un vecteur ${ }_{\alpha \beta}^{t r} \xi$ du type $\left(0,0 \ldots 0,{ }_{\alpha}^{t r} \pi, 0, \ldots 0,-{ }_{\beta}^{t r} \pi, 0 \ldots 0\right)$ si on veut comparer les deux sous-populations $\alpha$ et $\beta$ sur la base de la valeur de l'ensemble des animaux naissant. Par conséquent, si $e$ peut être écrit comme la somme d'un terme constant pour tous les groupes $(\mu)$ et d'un terme variable $(e=1$ $\mu+\mathrm{E})$, on pourra négliger $\mu: d e=t r \xi . e=t r \xi . \mathrm{E}$.

\section{a) Populations de type I}

On s'intéresse ici aux comparaisons du type $d e_{i j}=t r \xi_{i j}$. E.

Avec les notations introduites dans la partie $I$, on peut écrire $(I-T)^{-1}$ sous la forme :

$$
(\mathrm{I}-\mathrm{T})^{-1}=(\mathrm{I}-\mathrm{P}+\mathrm{R})^{-1}=(\mathrm{I}-\mathrm{P}+\mathrm{M}-(\mathrm{M}-\mathrm{R}))^{-1}=(\mathrm{I}-\mathrm{H}(\mathrm{M}-\mathrm{R}))^{-1} \mathrm{H}
$$

donc:

$$
\begin{aligned}
(\mathrm{I}-\mathrm{T})^{-1} & =\mathrm{H}+\mathrm{H} \cdot(\mathrm{M}-\mathrm{R}) \cdot(\mathrm{I}-\mathrm{T})^{-1} \\
& =\mathrm{H}+\mathrm{H} \cdot(\mathrm{M}-\mathrm{R}) \cdot \mathrm{H}+\mathrm{H} \cdot(\mathrm{M}-\mathrm{R}) \cdot \mathrm{H} \cdot(\mathrm{M}-\mathrm{R}) \cdot(\mathrm{I}-\mathrm{T})^{-1}
\end{aligned}
$$




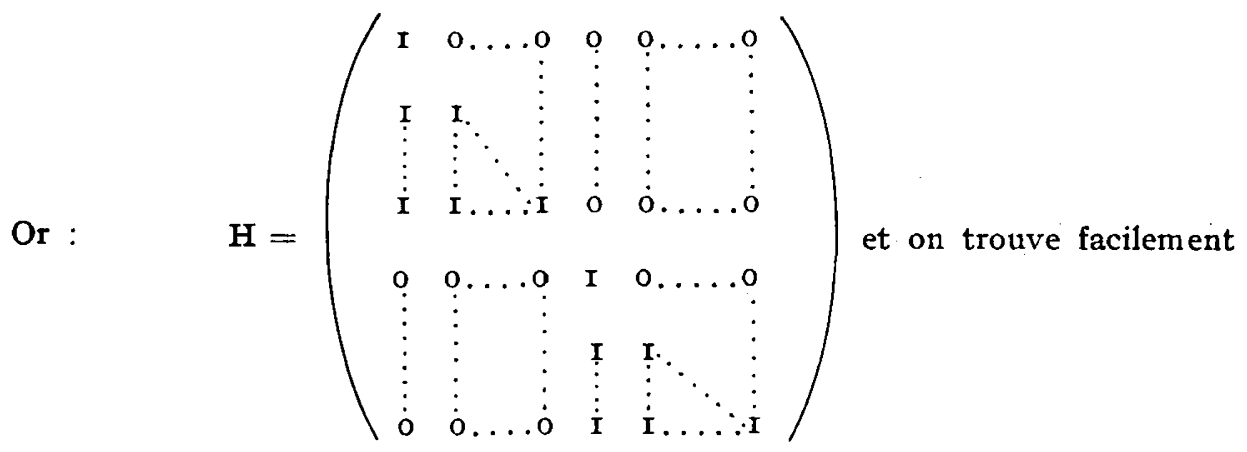

que HMHM est une matrice dont toutes les lignes sont identiques au vecteur

$$
\left(\frac{{ }^{\operatorname{tr}} a+{ }^{t r} c}{2}, \frac{{ }^{\operatorname{tr}} b+{ }^{t r} d}{2}\right)
$$

Alors, $(\mathrm{I}-\mathrm{T})^{-1}-\mathrm{HMHM} .(\mathrm{I}-\mathrm{T})^{-1}=\mathrm{Z}=\mathrm{H}+\mathrm{HMH}-\mathrm{HR}(\mathrm{H}+$

$$
\mathrm{H} \cdot(\mathrm{M}-\mathrm{R}) \cdot(\mathrm{I}-\mathrm{T}))^{-1}-\mathrm{HMHR} \cdot(\mathrm{I}-\mathrm{T})^{-1}
$$

Soit, puisque $\mathrm{R}(\mathrm{I}-\mathrm{T})^{-1}=\mathrm{R}$

$$
\begin{aligned}
Z & =(\mathrm{H}+\mathrm{HMH})-\mathrm{HR} \cdot(\mathrm{I}-\mathrm{T})^{-1}-\mathrm{HMHR} \cdot(\mathrm{I}-\mathrm{T})^{-1} \\
& =\mathrm{H} \cdot(\mathrm{I}+\mathrm{MH}) \cdot(\mathrm{I}-\mathrm{R})
\end{aligned}
$$

et les écarts $d e_{i j}$ sont donnés par ${ }^{t r} \xi_{i j} . Z . \mathrm{V}$

soit $d e_{i j}={ }^{t r} \xi_{i j}(\mathrm{H}+\mathrm{HMH})(\mathrm{V}-1 . \mathrm{G})$

$\mathrm{HMH}$ est une matrice dont les $n$ premières lignes sont $\left({ }^{t r} \mathrm{~A},{ }^{t r} \mathrm{~B}\right)$ et les $m$ suivantes $\left({ }^{t r} \mathrm{C},{ }^{t r} \mathrm{D}\right)$.

Alors en notant que ${ }^{t r} \mathrm{~A} .1 n,{ }^{t r} \mathrm{~B} .1 \mathrm{~m},{ }^{t r} \mathrm{C} .1 n,{ }^{t r} \mathrm{D} .1 n$ sont la moitié des inter-

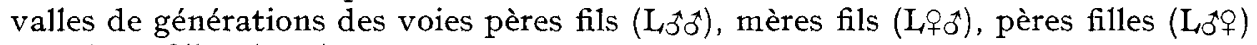
et mères filles (L웅) ,

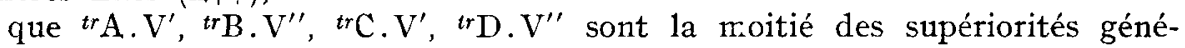

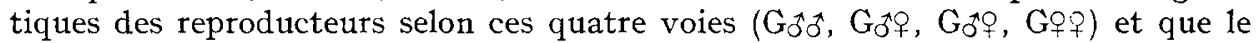
progrès annuel

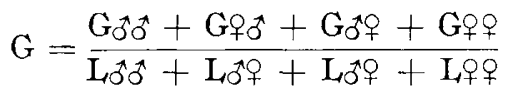

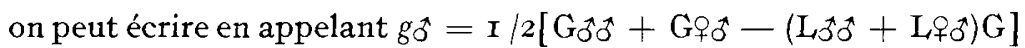

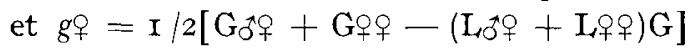

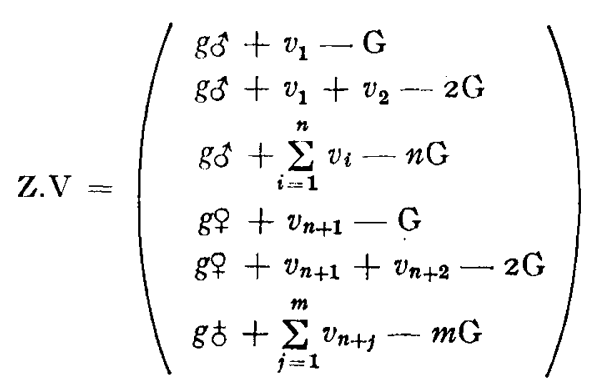


Donc l'écart entre deux groupes de mâles $i$ et $j$ est :

$$
\sum_{k=i+1}^{i} v_{k}+(i-j) \mathrm{G}
$$

et l'écart entre mâles et femelles de même âge $i$ :

$$
\sum_{k=1}^{i}\left(v_{k}-v_{n+k}\right)+2 g \text { ๙ }=\sum_{k=1}^{i}\left(v_{k}-v_{n+k}\right)-2 g \text { \% }
$$

On peut par ailleurs obtenir une formule sans inversion de matrice pour le vecteur des écarts $e=(\mathrm{I}-\mathrm{T})^{-1} \mathrm{~V}$ si on note que $\mathrm{T}(\mathrm{I}-\mathrm{T})^{-1}=(\mathrm{I}-\mathrm{T})^{-1}$ - I et que T. HMHM $=0$.

En effet, en prémultipliant par 'T l'égalité $\left(\mathrm{I}-\mathrm{T}^{\mathrm{T}}\right)^{-1}=Z+\operatorname{HMHM}(\mathrm{I}-\mathrm{T})^{-1}$, on trouve que $(\mathrm{I}-\mathrm{T})^{-1}=\mathrm{I}+\mathrm{T} . \mathrm{Z}$

donc que $e=\mathrm{V}+\mathrm{T}$. E

b) Populations de type 2

La matrice $(\mathrm{I}-\mathrm{T})^{-1}=(\mathrm{I}-\mathrm{P}+\mathrm{R})^{-1}$ est ici :

$$
\left(\begin{array}{cc}
\mathrm{I}-{ }_{11} \mathrm{P}+{ }_{11} \mathrm{R} & 0 \\
-{ }_{21} \mathrm{P}+{ }_{21} \mathrm{R} & \mathrm{I}-{ }_{22} \mathrm{P}
\end{array}\right)^{-1}
$$

les lignes de ${ }_{11} \mathrm{R}$ et ${ }_{21} \mathrm{R}$ étant celle de ${ }_{11} \mathrm{P}^{\infty}$.

Comme précédemment nous alons définir des matrices $\mathrm{N}$ et $\mathrm{K}=$ $(I-P+N)^{-1}$ de sorte que $(I-T)^{-1}$ puisse s'écrire $(I-K(N-R))^{-1} K$. Nous choisirons $\mathrm{N}=\left(\begin{array}{rr}{ }_{11} \mathrm{M} & 0 \\ 0 & 0\end{array}\right),{ }_{11} \mathrm{M}$ étant définie comme plus haut pour la souspopulation $\mathrm{I}$.

$\mathrm{A}_{11} \mathrm{M}$ correspond ${ }_{11} \mathrm{H}=\left(\mathrm{I}-{ }_{11} \mathrm{P}+{ }_{11} \mathrm{M}\right)^{-1}$. Avec cette définition de $\mathrm{K}$, on retrouve que KNKN est formée de lignes toutes identiques.

En effet :

$$
\mathrm{K}=\left(\begin{array}{cc}
\mathrm{I}-{ }_{11} \mathrm{P}+{ }_{11} \mathrm{M} & \mathrm{O} \\
-{ }_{21} \mathrm{P} & \mathrm{I}-{ }_{22} \mathrm{P}
\end{array}\right)^{-1}
$$

Soit

$$
\mathrm{K}=\left(\begin{array}{l}
{ }_{11} \mathrm{H} \\
\left(\mathrm{I}-{ }_{22} \mathrm{P}\right)^{-1} \cdot{ }_{21} \mathrm{P} \cdot{ }_{11} \mathrm{H} \quad\left(\mathrm{I}-{ }_{22} \mathrm{P}\right)^{-1}
\end{array}\right)
$$

et

$$
\mathrm{KNKN}=\left(\begin{array}{ll}
{ }_{11} \mathrm{H} \cdot{ }_{11} \mathrm{M} \cdot{ }_{11} \mathrm{H} \cdot{ }_{11} \mathrm{M} & \mathrm{O} \\
\left(\mathrm{I}-{ }_{22} \mathrm{P}\right)^{-1} \cdot{ }_{21} \mathrm{P} \cdot{ }_{11} \mathrm{H} \cdot{ }_{11} \mathrm{M} \cdot{ }_{11} \mathrm{H} \cdot{ }_{11} \mathrm{M} & \mathrm{o}
\end{array}\right)
$$

Or $\left(\mathrm{I}-{ }_{22} \mathrm{P}\right)^{-1} \cdot{ }_{21} \mathrm{P} .1=1$, donc toutes les lignes de $\mathrm{KNKN}$ sont identiques à

$$
\left(\frac{{ }_{11}^{t r} a+{ }_{11}^{t r} c}{2}, \frac{{ }_{11}^{t r} b+{ }_{11}^{t r} d}{2}, 0,0\right)
$$

Le vecteur des écarts $\mathrm{E}$ s'écrit donc encore $\mathrm{E}=\mathrm{K}$. (I $+\mathrm{NK}) .(\mathrm{I}-\mathrm{R})$. V. $\mathrm{Si}$ on revient à la décomposition de $\mathrm{P}$ en sous-matrices élémentaires :

$$
P=\left(\begin{array}{rrr}
{ }_{11} Q & O & O \\
{ }_{21} Q & { }_{22} Q & O \\
O & { }_{32} Q & { }_{33} Q
\end{array}\right)
$$


on a :

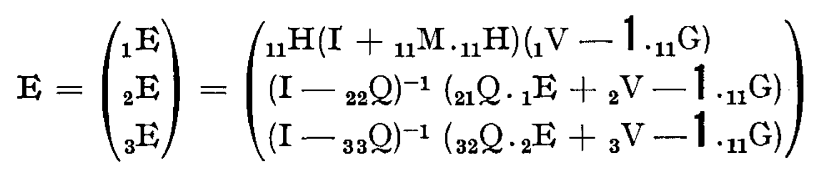

Le noyau se comporte donc comme une population fermée de type I. L'expression des écarts ${ }_{3} \mathrm{E}$ pour la base est plus complexe.

On les écrira ${ }_{\varepsilon} \mathrm{E}={ }_{3} \mathrm{E}-{ }_{2} \mathrm{E}+{ }_{2} \mathrm{E}-{ }_{1} \mathrm{E}+{ }_{1} \mathrm{E},{ }_{3} \mathrm{E}-{ }_{1} \mathrm{E}$ étant d'ailleurs le vecteur des décalages entre le noyau et la base pour des reproducteurs de même âge et sexe. En pratique, plus que les écarts intra sous-population, c'est ici l'écart entre le noyau et la base qui nous intéresse. Cet écart est donné par :

$$
d e={ }_{2}^{t r}{ }_{2} \mathrm{E}+{ }^{t r} \pi \cdot{ }_{3} \mathrm{E}-{ }_{1}^{t r}{ }_{\cdot 1} \mathrm{E}
$$

et correspond au " time-lag " étudié par BICHARD (I97I).

On a alors ${ }_{2} \mathrm{E}-{ }_{1} \mathrm{E}=\left(\mathrm{I}-{ }_{22} \mathrm{Q}\right)^{-1} \cdot\left[\left({ }_{21} \mathrm{Q}+{ }_{22} \mathrm{Q}-\mathrm{I}\right){ }_{{ }_{1}} \mathrm{E}+{ }_{2} \mathrm{~V}-1 \cdot{ }_{11} \mathrm{G}\right]$ où $\left(\mathrm{I}-{ }_{22} \mathrm{Q}\right)^{-1}={ }_{22} \mathrm{H}$. On notera ${ }_{2} \mathrm{Q}={ }_{21} \mathrm{Q}+{ }_{22} \mathrm{Q}$.

$\mathrm{Si}_{22} m$ et ${ }_{22} f$ sont les vecteurs

$$
{ }_{22} m_{2}=\left(\begin{array}{l}
\mathrm{I} \\
{ }_{22} m_{2} \\
{ }_{22} m_{2} \cdot{ }_{22} m_{3} \\
{ }_{22} m_{2} \ldots \ldots{ }_{22} m_{n}
\end{array}\right) \quad{ }_{22} t=\left(\begin{array}{l}
\mathrm{I} \\
{ }_{22} f_{2} \\
{ }_{22} f_{2} \cdot{ }_{22} f_{3} \\
{ }_{22} f_{2} \ldots{ }_{22} f_{m}
\end{array}\right)
$$

on trouve que,

$$
{ }_{2} \mathrm{E}-{ }_{1} \mathrm{E}=\left(\begin{array}{l}
{ }_{22} m \cdot\left({ }_{21} g \hat{\gamma}-{ }_{11} g \sigma^{\uparrow}\right) \\
{ }_{22} f \cdot\left({ }_{21} g \text { 우 }-{ }_{11} g \text { ㅇ }\right)
\end{array}\right)+{ }_{22} \mathrm{H}\left({ }_{2} \mathrm{~V}-{ }_{1} \mathrm{~V}\right)
$$

${ }_{21} g \widehat{\partial}$ et ${ }_{21} g$ ㅇ étant définis de façon similaire à ${ }_{11} g \sigma^{\star}$ et ${ }_{11} g$ ㅇ par

et

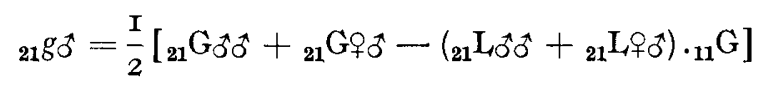

$$
\begin{aligned}
& 2 \mathrm{I} g \text { 우 }=\frac{\mathrm{I}}{2}\left[{ }_{21} \mathrm{G} \text { \%우 }+{ }_{21} \mathrm{G} \text { 우 }-\left({ }_{21} \mathrm{~L} \text { 웅 }+{ }_{21} \mathrm{~L} \text { 웅 }\right) \cdot{ }_{11} \mathrm{G}\right]
\end{aligned}
$$

De la même façon ${ }_{3} \mathrm{E}-{ }_{2} \mathrm{E}=\left(\mathrm{I}-{ }_{33} \mathrm{Q}\right)^{-1}\left[\left({ }_{32} \mathrm{Q}+{ }_{33} \mathrm{Q}-\mathrm{I}\right) \cdot{ }_{2} \mathrm{E}+{ }_{3} \mathrm{~V}\right.$ $\left.-1 \cdot{ }_{11} \mathrm{G}\right]$. Soit $\mathrm{W}$ le vecteur $\left({ }_{32} \mathrm{Q}+{ }_{33} \mathrm{Q}-\mathrm{I}\right){ }_{2} \mathrm{E}+{ }_{3} \mathrm{~V}-1 \cdot{ }_{11} \mathrm{G}$; la différence ${ }_{3} \mathrm{E}-{ }_{2} \mathrm{E}$ est obtenue à partir de l'expression de la matrice $\left(\mathrm{I}-{ }_{33} Q\right)^{-1}$ donnée en annexe $I$. $S i E_{0}^{\prime}$ et $E_{0}^{\prime \prime}$ sont :

$$
\begin{aligned}
& \mathrm{E}_{0}^{\prime}=\frac{\mathrm{I}}{{ }_{33} \delta}\left[\left(\mathrm{I}-{ }_{33} \mathrm{D}_{1}\right) \cdot\left[{ }_{33}^{t r} \mathrm{~A} \cdot \mathrm{W}^{\prime}+{ }_{33}^{t r} \mathrm{~B} \cdot \mathrm{W}^{\prime \prime}\right]+{ }_{33} \mathrm{~B} \cdot \cdot\left[{ }_{33}^{t r} \mathrm{C} \cdot \mathrm{W}^{\prime}+{ }_{33}^{t r} \mathrm{D} \cdot \mathrm{W}^{\prime \prime}\right]\right] \\
& \mathrm{E}_{0}^{\prime \prime}=\frac{\mathrm{I}}{{ }_{33} \delta}\left[\left(\mathrm{I}-{ }_{33} \mathrm{~A}_{1}\right) \cdot\left[{ }_{33}^{t r} \mathrm{C} \cdot \mathrm{W}^{\prime}+{ }_{33}^{t r} \mathrm{D} \cdot \mathrm{W}^{\prime \prime}\right]+{ }_{33} \mathrm{C} \cdot\left[\cdot\left[{ }_{33}^{t r} \mathrm{~A} \cdot \mathrm{W}^{\prime}+{ }_{33}^{t r} \mathrm{~B} \cdot \mathrm{W}^{\prime \prime}\right]\right]\right.
\end{aligned}
$$

alors $\quad{ }_{3} \mathrm{E}-{ }_{2} \mathrm{E}=\left(\begin{array}{l}1_{n} \cdot \mathrm{E}_{0}^{\prime} \\ 1_{m} \cdot \mathrm{E}_{0}^{\prime \prime}\end{array}\right)+{ }_{33} \mathrm{H} \cdot \mathrm{W}$

Donc le $i$ ème élément de ${ }_{3} \mathrm{E}-{ }_{2} \mathrm{E}$ est $\mathrm{E}_{0}^{\prime}+\sum_{k=1}^{i} w_{k}$ si $i \leqslant n$

$$
\mathrm{E}_{0}^{\prime \prime}+\sum_{k=1}^{i} w_{n+k} \text { si } i>n
$$


Dans cette situation générale il est difficile d'aller plus loin dans l'expression des écarts. On peut essayer de retrouver les résultats de Bichard (r97I) en se plaçant sous les hypothèses formulées ou implicites qu'il faisait et en particulier 1'identité des deux voies pères fils et pères filles et des deux voies mères fils et mères filles (hypothèse Ho).

Outre la possibilité de sélectionner les reproducteurs de la base, BICHARD (I97I) a envisagé deux méthodes pour jouer sur l'écart entre les sous-populations : utiliser des reproducteurs nés dans la base et récupérer pour la base des animaux s'étant déjà reproduits dans le noyau. Nous n'envisagerons ici que les situations où seule la première option est réalisée.

Dans ce cas, avec nos notations, ${ }_{21} \mathrm{Q}={ }_{21} \mathrm{M}$

donc ${ }_{2} \mathrm{E}=1 .{ }_{21} g+{ }_{22} \mathrm{H}\left({ }_{2} \mathrm{~V}-1 \cdot{ }_{11} \mathrm{G}\right) \quad{ }_{21} g={ }_{21} g \hat{o}={ }_{21} g \circ$ puisqu'on suppose Ho réalisé.

et $\mathrm{W}$ est le vecteur :

$$
\mathrm{W}=\left(\begin{array}{c}
32 g \hat{\sigma} \\
0 \\
0 \\
32 g q \\
0 \\
0
\end{array}\right)+{ }_{3} \mathrm{~V}-{ }_{2} \mathrm{~V}
$$

avec

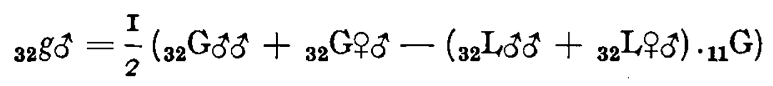

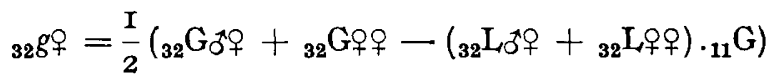

Nous allons maintenant supposer avec BICHARD (I97I) que la proportion de pères (ou de mères) des reproducteurs de la base qui sont nés dans la base est indépendante de leur âge (hypothèse $\mathrm{Hr}$ ), soit que :

$$
\begin{aligned}
& { }_{33} a_{i}=\lambda_{a}\left({ }_{32} a_{i}+{ }_{33} a_{i}\right) \text { pour tout } i \\
& { }_{33} b_{j}=\lambda_{b}\left({ }_{32} b_{j}+{ }_{{ }_{3}} b_{j}\right) \\
& { }_{33} c_{i}=\lambda_{c}\left({ }_{32} c_{i}+{ }_{33} c_{i}\right) \\
& { }_{33} d_{j}=\lambda_{d}\left({ }_{32} d_{j}+{ }_{33} d_{j}\right)
\end{aligned}
$$

Sous cette hypothèse en effet,

et

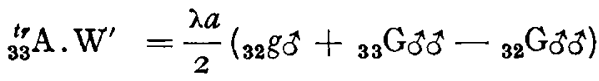

$$
\begin{aligned}
& { }_{33}^{t r} \mathrm{~B} \cdot \mathrm{W}^{\prime \prime}=\frac{\lambda b}{2}\left({ }_{32} g q+{ }_{33} \mathrm{G} q 0^{\star}-{ }_{32} \mathrm{G}+\sigma^{\lambda}\right) \\
& { }_{33}^{t r} \mathrm{C} \cdot \mathrm{W}^{\prime}=\frac{\lambda c}{2}\left({ }_{32} g \delta^{\prime}+{ }_{33} \mathrm{G} \tilde{\delta}^{\prime} q-{ }_{32} \mathrm{G} \delta^{\prime}+\right) \\
& { }_{33}^{t r} \mathrm{D} \cdot \mathrm{W}^{\prime \prime}=\frac{\lambda d}{2}\left({ }_{32} g q+{ }_{33} \mathrm{G} q q-{ }_{32} \mathrm{G} q q\right) \\
& { }_{3} \mathrm{E}-{ }_{3} \mathrm{E}=\left(\begin{array}{l}
1 n \cdot \mathrm{K}_{0}^{\prime} \\
1 m \cdot \mathrm{K}_{0}^{\prime \prime}
\end{array}\right)+{ }_{33} \mathrm{H}\left({ }_{3} \mathrm{~V}-{ }_{2} \mathrm{~V}\right)
\end{aligned}
$$


avec :

$$
\begin{aligned}
& \mathrm{K}_{0}^{\prime}\left(\left(\mathrm{I}-\frac{\lambda a}{2}\right) \cdot\left(\mathrm{I}-\frac{\lambda d}{2}\right)-\frac{\lambda b}{2} \frac{\lambda c}{2}\right)=\left(\mathrm{I}-\frac{\lambda d}{2}\right) \cdot{ }_{32} g \partial+\frac{\lambda b}{2} \cdot{ }_{32} g \rho+
\end{aligned}
$$

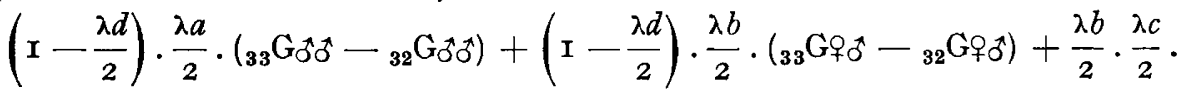

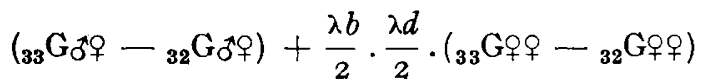

et :

$$
\begin{aligned}
& \mathbf{K}_{0}^{\prime \prime}\left(\left(\mathrm{I}-\frac{\lambda a}{2}\right) \cdot\left(\mathrm{I}-\frac{\lambda d}{2}\right)-\frac{\lambda b}{2} \cdot \frac{\lambda c}{2}\right)=\frac{\lambda c}{2} \cdot{ }_{32} g \sigma^{\dagger}+\left(\mathrm{I}-\frac{\lambda a}{2}\right) \cdot{ }_{32} g \rho+\left(\mathrm{I}-\frac{\lambda a}{2}\right) \cdot \frac{\lambda c}{2} .
\end{aligned}
$$

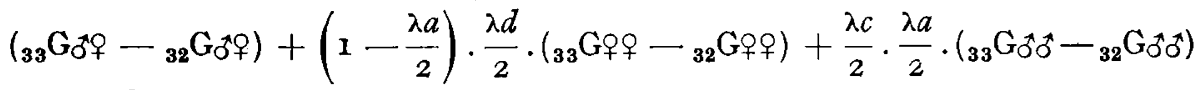

$$
\begin{aligned}
& +\frac{\lambda c}{2} \cdot \frac{\lambda b}{2} \cdot\left({ }_{33} \mathrm{G}+\sigma^{\lambda}-{ }_{32} \mathrm{G}+\sigma^{\lambda}\right) \text {. }
\end{aligned}
$$

On peut alors retrouver les formules établies par BICHARD (I97I)

- formule $7 a$ : tous les mâles viennent du noyau, toutes les femelles de la base.

On a ici $\lambda c=0 \quad \lambda d=\mathrm{I} \quad{ }_{2}^{t r} \pi=\left({ }_{2}^{t r} \pi^{\prime}, 0\right)$ et ${ }_{3}^{t r} \pi=\left(0,{ }_{3}^{t r} \pi^{\prime \prime}\right)$

donc $\mathrm{K}_{0}^{\prime \prime}=2 \cdot{ }_{32} g$ g $+\left({ }_{33} \mathrm{G} q\right.$ \% $\left.-{ }_{32} \mathrm{G} q q\right)$

$$
\begin{aligned}
& \text { et } d e={ }_{33} g \text { ㅇ }+\frac{{ }_{33} \mathrm{G} \text { 우 }-{ }_{32} \mathrm{G} \text { 우 }}{2}+{ }_{21} g+{ }_{13}{ }_{3} \pi \cdot{ }_{33} \mathrm{H} \cdot\left({ }_{3} \mathrm{~V}-1 \cdot{ }_{11} \mathrm{G}\right) \\
& \left.+{ }_{2}^{t r} \pi \cdot{ }_{2} \mathrm{H} \cdot{ }_{2} \mathrm{~V}-1 \cdot{ }_{11} \mathrm{G}\right)-{ }_{1}^{t r} \pi \cdot{ }_{11} \mathrm{H} \cdot\left({ }_{1} \mathrm{~V}-1 \cdot{ }_{11} \mathrm{G}\right)
\end{aligned}
$$

Or BICHARD suppose implicitement que tout animal né est candidat à la sélection (hypothèse $\mathbf{H 2}$ ) donc que ${ }_{3} \pi^{\prime \prime}={ }_{33} d,{ }_{2} \pi^{\prime}={ }_{32} a$ et ${ }_{1} \pi=\left({ }_{11} a,{ }_{11} b\right)=\left({ }_{11} c,{ }_{11} d\right)$ et que la pyramide des âges des parents des reproducteurs de la base nés dans le noyau est la même que celle des parents des reproducteurs du noyau (hypothèse $\mathbf{H}_{3}$ ) donc que $\mathrm{M}_{21}=\mathrm{M}_{11}$, ce qui donne ${ }_{21} \mathrm{~g}=0$.

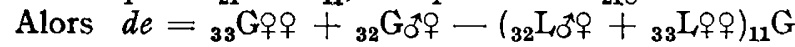

- formule Ir. Tous les reproducteurs viennent du noyau.

On a ici $\lambda a=\lambda b=\lambda c=\lambda d=0 \quad{ }_{3}^{t r} \pi=0$

donc : $\quad d e={ }_{2}^{t r} \cdot{ }_{2} \mathrm{E}-{ }_{1}^{t r} \pi \cdot{ }_{1} \mathrm{E}$

soit $\quad d e={ }_{21} g+{ }_{2}^{t r} \cdot{ }_{22} \mathrm{H} \cdot\left({ }_{2} \mathrm{~V}-1 \cdot{ }_{11} \mathrm{G}\right)-{ }^{t r} \pi \cdot{ }_{11} \mathrm{H} \cdot\left({ }_{1} \mathrm{~V}-1 \cdot{ }_{11} \mathrm{G}\right)$

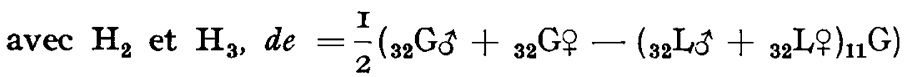

- formule $6 a$. Toutes les femelles viennent de la base, seule une fraction $d$ des mâles vient du noyau.

On a ici $\lambda a=\lambda c=\mathrm{I}-d, \lambda b=\lambda d=\mathrm{I}$

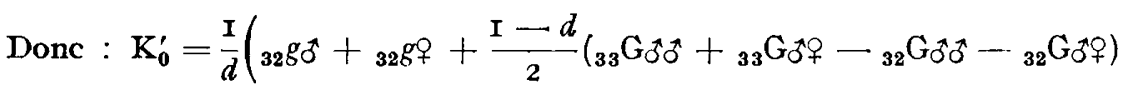

$$
+\frac{\mathrm{I}}{2}\left({ }_{33} \mathrm{G} q 0^{*}+{ }_{33} \mathrm{G}+q \text { - }-{ }_{32} \mathrm{G}+0^{*}-{ }_{32} \mathrm{G}+q \text { ) }\right)
$$




$$
\begin{aligned}
& \mathrm{K}_{0}^{\prime \prime}=\frac{\mathrm{I}}{d}\left((\mathrm{I}-d)_{32} g \sigma^{\star}+(\mathrm{I}+d)_{32} g \text { ㅇ }+\frac{(\mathrm{I}+d)(\mathrm{I}-d)}{2}\left({ }_{33} \mathrm{G}_{\sigma^{*}}+{ }_{32} \mathrm{G}_{0}{ }^{\circ}+\right)\right.
\end{aligned}
$$

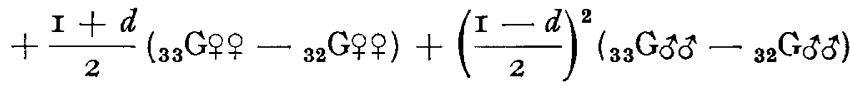

$$
\begin{aligned}
& \left.+\frac{\mathrm{I}-d}{2}\left({ }_{33} \mathrm{G}_{q 0}{ }^{*}-{ }_{32} \mathrm{G}+O^{*}\right)\right)
\end{aligned}
$$

Mais, avec Ho, on trouve que :

$$
\begin{aligned}
& \mathrm{K}_{0}^{\prime}=\mathrm{K}_{0}^{\prime \prime}=\frac{\mathrm{I}}{d}\left(2 \cdot{ }_{32} g+(\mathrm{I}-d)\left({ }_{33} \mathrm{G} \delta^{\star}-{ }_{32} \mathrm{G}_{\delta^{\prime}}\right)+{ }_{33} \mathrm{G} q-{ }_{32} \mathrm{G} q\right) \\
& =\frac{\mathrm{I}}{d}\left(2 \cdot{ }_{33} g-d\left({ }_{33} \mathrm{G}_{\circlearrowleft} \mathrm{O}-{ }_{32} \mathrm{G}_{\sigma^{*}}\right)\right)
\end{aligned}
$$

D'autre part $\mathrm{H}_{2}$ implique ${ }_{2} \pi^{\prime}={ }_{32} a={ }_{32} c=d\left({ }_{2} \pi^{\prime}+{ }_{3} \pi^{\prime}\right)$

$$
\begin{aligned}
{ }_{3} \pi^{\prime} & ={ }_{33} a={ }_{33} c=(\mathrm{I}-d)\left({ }_{2} \pi^{\prime}+{ }_{3_{3}} \pi^{\prime}\right) \\
\text { et }{ }_{2} \pi^{\prime \prime} & =0
\end{aligned}
$$

et, sous $\mathrm{H}_{3}$, on peut écrire :

$$
\begin{aligned}
& { }_{2}^{t r} \pi \cdot{ }_{2} \mathrm{E}={ }_{2}^{t r} \pi^{\prime} \cdot{ }_{2} \mathrm{E}^{\prime}=\frac{d}{2}\left({ }_{32} \mathrm{G} \hat{O}-{ }_{32} \mathrm{~L} \hat{O}^{\hat{0}} \cdot{ }_{11} \mathrm{G}\right) \\
& { }_{3}^{t r} \pi^{\prime} \cdot{ }_{3} \mathrm{E}^{\prime}=\frac{\mathrm{I}-d}{2}\left(\mathrm{~K}_{0}^{\prime}+{ }_{33} \mathrm{G} \sigma^{*}-{ }_{33} \mathrm{~L}_{0}{ }^{\prime} \cdot{ }_{11} \mathrm{G}\right) \\
& { }_{3}^{t r} \pi^{\prime \prime} \cdot{ }_{3} \mathrm{E}^{\prime \prime}=\frac{\mathrm{I}}{2}\left(\mathrm{~K}_{0}^{\prime \prime}+{ }_{33} \mathrm{G} q-{ }_{33} \mathrm{~L} \text { 우 }{ }_{11} \mathrm{G}\right)
\end{aligned}
$$

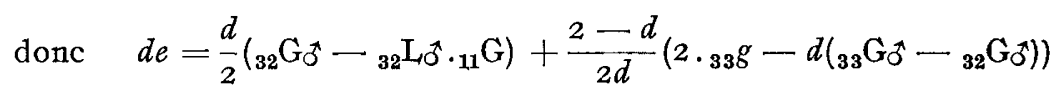

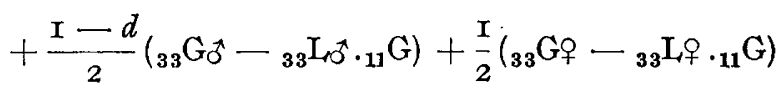

soit $\quad d e={ }_{33} g\left(\frac{2-d}{d}-\mathrm{I}\right)+\left({ }_{33} \mathrm{G} q-{ }_{33} \mathrm{~L} q_{11} \mathrm{G}\right)+\left({ }_{32} \mathrm{G}_{0}^{*}-{ }_{32} \mathrm{~L}{ }_{11} \mathrm{G}\right)\left(\frac{d}{2}+\frac{2-d}{2}\right)$

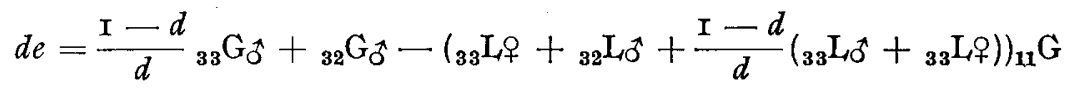

En conclusion, les formules données plus haut sont une généralisation des résultats de BICHARD (I97I) permettant non seulement de prendre en compte le chevauchement des générations mais aussi de distinguer entre les quatre voies de transmission du progrès génétique $(\mathrm{Ho})$, d'envisager des pyramides des âges différant selon l'origine et la destination des reproducteurs $\left(\mathrm{H}_{3}\right)$, de traiter le cas de modification de l'utilisation avec l'âge (HI) et de tenir compte d'un éventuel choix sur ascendance des candidats à la sélection $\left(\mathrm{H}_{2}\right)$.

c) Populations de type 3 et 5

Nous ne donnerons pas de résultats détaillés pour ces populations, la démarche restant la même : on écrira $(I-T)^{-1}$ sous la forme $\left(I-K(N-R)^{-1}\right) K$ et le vecteur des écarts pourra être ramené à $\mathrm{E}=\mathrm{K}(\mathrm{I}+\mathrm{NK})(\mathrm{I}-\mathrm{R}) \mathrm{V}$. 
Dans le cas de ces populations la matrice $\mathrm{P}$ s'écrit :

$$
P=\left(\begin{array}{rrrrrr}
{ }_{11} Q & O & O & O & O & O \\
{ }_{21} Q & { }_{22} Q & 0 & O & 0 & O \\
Q & { }_{32} Q & { }_{33} Q & O & 0 & O \\
{ }_{41} Q & O & O & { }_{44} Q & O & O \\
O & O & { }_{53} Q & O & { }_{55} Q & O \\
O & O & O & { }_{64} Q & { }_{65} Q & { }_{66} Q
\end{array}\right)
$$

et les écarts sont donnés par les vecteurs :

- reproducteurs du noyau :

$$
{ }_{1} \mathrm{E}={ }_{11} \mathrm{H} \cdot\left(\mathrm{I}+{ }_{11} \mathrm{M} \cdot{ }_{11} \mathrm{H}\right)\left({ }_{1} \mathrm{~V}-1_{11} \mathrm{G}\right)
$$

- reproducteurs de la multiplication nés dans le noyau :

$$
{ }_{2} \mathrm{E}=\left(\mathrm{I}-{ }_{22} \mathrm{Q}\right)^{-1}\left({ }_{21} \mathrm{Q} \cdot{ }_{1} \mathrm{E}+{ }_{2} \mathrm{~V}-1_{11} \mathrm{G}\right)
$$

- reproducteurs de la multiplication nés dans la multiplication :

$$
{ }_{3} \mathrm{E}=\left(\mathrm{I}-{ }_{33} \mathrm{Q}\right)^{-1}\left({ }_{32} \mathrm{Q} \cdot{ }_{2} \mathrm{E}+{ }_{3} \mathrm{~V}-1_{11} \mathrm{G}\right)
$$

- reproducteurs de la base nés dans le noyau :

$$
{ }_{4} \mathrm{E}=\left(\mathrm{I}-{ }_{44} \mathrm{Q}\right)^{-1}\left({ }_{41} \mathrm{Q} \cdot{ }_{1} \mathrm{E}+{ }_{4} \mathrm{~V}-1_{11} \mathrm{G}\right)
$$

- reproducteurs de la base nés dans la multiplication :

$$
{ }_{5} \mathrm{E}=\left(\mathrm{I}-{ }_{55} \mathrm{Q}\right)^{-1}\left({ }_{53} \mathrm{Q} \cdot{ }_{3} \mathrm{E}+{ }_{5} \mathrm{~V}-1_{11} \mathrm{G}\right)
$$

- reproducteurs de la base nés dans la base :

$$
{ }_{6} \mathrm{E}=\left(\mathrm{I}-{ }_{66} \mathrm{Q}\right)^{-1}\left({ }_{64} \mathrm{Q} \cdot{ }_{4} \mathrm{E}+{ }_{65} \mathrm{Q} \cdot{ }_{5} \mathrm{E}+{ }_{6} \mathrm{~V}-1_{11} \mathrm{G}\right)
$$

ou ${ }_{11} \mathrm{H}$ et ${ }_{11} \mathrm{M}$ sont définis comme en $\mathrm{II}_{3} b$.

\section{d) Populations de type 4}

Pour les populations croisées la notion de décalage telle qu'elle était 'définie par BICHARD (197I) perd son sens dans la mesure où les deux noyaux ne progressent pas nécessairement à la même vitesse pour un même caractère.

S'il est toujours possible de calculer les écarts intra-noyau entre groupes d'animaux en appliquant directement les formules données en II $3 a$, ni la signification, ni une méthode simple de calcul des écarts entre sous-populations ne nous sont apparues clairement. On peut cependant en revenant à la définition initiale écrire :

$$
\begin{aligned}
& (\mathrm{I}-\mathrm{T})^{-1}= \\
& \left(\begin{array}{ccc}
\left(\mathrm{I}-{ }_{11} \mathrm{~T}\right)^{-1} & 0 & 0 \\
0 & \left(\mathrm{I}-{ }_{22} \mathrm{~T}\right)^{-1} & 0 \\
\left(\mathrm{I}-{ }_{33} \mathrm{P}\right)^{-1}\left({ }_{31} \mathrm{P}-{ }_{31} \mathrm{R}\right)\left(\mathrm{I}-{ }_{11} \mathrm{~T}\right)^{-1} & \left(\mathrm{I}-{ }_{33} \mathrm{P}\right)^{-1}\left({ }_{32} \mathrm{P}-{ }_{32} \mathrm{R}\right)\left(\mathrm{I}-{ }_{22} \mathrm{~T}\right)^{-1} & \left(\mathrm{I}-{ }_{33} \mathrm{P}\right)^{-1}
\end{array}\right)
\end{aligned}
$$


et on a

${ }_{1} e=\left(\mathrm{I}-{ }_{11} \mathrm{~B}\right)^{-1} \cdot{ }_{1} \mathrm{~V}$

${ }_{2} e=\left(\mathrm{I}-{ }_{22} \mathrm{~B}\right)^{-1} \cdot{ }_{2} \mathrm{~V}$

${ }_{3} e=\left(\mathrm{I}-{ }_{33} \mathrm{P}\right)^{-1} \cdot\left({ }_{31} \mathrm{P} \cdot{ }_{1} e+{ }_{32} \mathrm{P} \cdot{ }_{2} e+{ }_{3} \mathrm{~V}-1\left({ }_{11} \mathrm{G}+{ }_{12} \mathrm{G}\right)\right)$.

\section{III. - Résultats à court et moyen terme}

\section{I. - Généralités}

Les résultats obtenus jusqu'ici supposaient que le plan de sélection étart appliqué depuis un temps suffisamment long pour que le niveau génétique moyen de la population s'accroisse chaque année d'une valeur constante donnée par le vecteur RV. Cette situation, si elle est intéressante d'un point de vue théorique pour comparer rapidement des schémas de sélection sur leurs effets à long terme, n'est cependant pas la règle générale. En pratique, on est confronté à deux types de problèmes dont la solution fait appel à la même démarche :

— on met en place un plan de sélection et on veut en connaître l'ensemble des répercussions dans le temps (HII, I97I, Ei,SEN et SELLIER I979);

- on tente une analyse coût-bénéfice d'un schéma de sélection en routine en isolant les effets d'une année ou d'un cycle de sélection (ensemble des opérations d'amélioration génétique pratiquées sur tous les individus nés la même année) (HINKS, I97I).

Dans ces deux cas, en effet, il faudra être capable de mettre en relation les efforts de contrôle et de sélection effectués à des instants précis ave z leurs conséquences qui se manifesteront tant que la population existera. Dans la première optique ces conséquences sont données par la suite des vecteurs $\mathrm{X}(t)$ ou des scalaires $\mathrm{Y}(t)$ tels qu'ils ont été définis en II $\mathbf{I}$. type :

Il est désormais classique de résumer cette suite en une fonction recettes du

$$
\mathbf{r}_{2}=r \sum_{t=0}^{\tau} \theta^{t} \mathrm{Y}(t) \quad \text { ou } \quad \sum_{t=0}^{\tau} \theta^{t} \operatorname{tr}(\pi r) \mathrm{X}(t)
$$

$\theta$ étant un coefficient d'actualisation $\left(\theta=\frac{I}{I+\varepsilon}, \varepsilon\right.$ le taux d'actualisation) et $r$ un facteur constant dépendant des effectifs d'animaux exprimant le caractère amélioré et des marges unitaires de ces caractères.

En notant avec Cunningham et JoAn Ryan (I975) qu'au de I5-20 ans les recettes cumulées actualisées ne dépendent pratiquement plus de la durée d'actualisation, on pourra généralement choisir 1'horizon infini et écrire :

soit

$$
\mathbf{r}_{2}=\operatorname{tr}(\pi r) \sum_{t=0}^{\infty} \theta^{t} \mathrm{X}(t)
$$

$$
\begin{aligned}
\mathbf{r}_{2} & =\operatorname{tr}(\pi r) \sum_{t=0}^{\infty} \theta^{t}\left(\mathrm{P}^{t} \mathrm{X}(\mathrm{o})+\left(\mathrm{P}^{t+1}+\ldots+\mathrm{I}\right) \mathrm{V}\right) \\
& =\operatorname{tr}(\pi r)\left(\mathrm{I}-\theta \mathrm{P}^{-1}\left(\mathrm{X}(\mathrm{o})+\frac{\theta}{\mathrm{I}-\theta} \mathrm{V}\right)\right.
\end{aligned}
$$


On remarque alors que l'effet d'une année de sélection - critère recherché dans la $z^{\mathbf{e}}$ optique - peut s'écrire :

$$
\begin{aligned}
& \mathbf{r}_{1}=\theta^{\operatorname{tr}}(\pi r)(\mathrm{I}-\theta \mathrm{P})^{-1} \mathrm{~V} \text { et que si l'on fait abstraction du niveau initial, } \\
& \mathbf{r}_{2}=\frac{\mathrm{I}}{\mathrm{I}-\theta} \mathbf{r}_{1} \text {. }
\end{aligned}
$$

Ainsi, l'inversion de la matrice $\mathrm{I}-\theta \mathrm{P}$, qui est du type $\mathrm{I}-{ }_{\alpha \beta} \mathrm{Q}$, joue à nouveau un rôle central dans l'étude de la diffusion du progrès génétique.

Or, dans la recherche d'un plan d'amélioration génétique optimum quant à ses répercussions sur le niveau de la population relativement aux efforts de sélection consentis pour les obtenir, on est toujours conduit à tester un grand nombre de valeurs des variables caractérisant ce plan : on se donne un critère de comparaison $\Delta=f\left(\mathbf{r}_{1}, \mathbf{t}\right)$ fonction des recettes actualisées et des coûts $\mathbf{t}$ de sélection et on classe les différents plans possibles sur la base de la valeur espérée pour $\Delta$.

Ainsi, LINDHE (I 968) comparait-il ro 800 plans de sélection des taureaux mixtes sur leur taux de rentabilité en combinant les valeurs possibles de quatre variables, cependant que HuNT et al. (I974) ont calculé dans I 440 situations le gain génétique espéré pour un schéma d'amélioration de la production laitière.

En outre, il est nécessaire, pour comparer valablement les différentes options, de pratiquer une analyse de sensibilité du critère $\Delta$ aux variations des paramètres mal connus ou susceptibles de changer dans le temps.

Un aperçu des divers paramètres et variables généralement étudiés est donné au tableau 2. Il ressort de celui-ci que nombre d'entre eux interviennent dans l'expression de la matrice $P$ et donc de l'inverse $(I-\theta P)^{-1}$, ce qui peut être un gros handicap du fait du temps de calcul assez long nécessaire aux inversions de matrices.

Dès lors, on est tenté de rechercher l'expression directe de $(\mathrm{I}-\theta \mathrm{P})^{-\mathbf{1}}$.

\section{2. - Population de type I}

L'inverse $(\mathrm{I}-\theta \mathrm{P})^{-1}$ est donné en annexe $\mathrm{I}$ avec ici :

$$
\begin{aligned}
& m_{i}=\theta \text { et } f_{j}=\theta \text { pour tout } i \text { et } i \\
& \mathrm{~A}_{i}=\theta a_{i}+\theta^{2} a_{i+1}+\theta^{3} a_{i+2}+\ldots+\theta^{n-i+1} a_{n}
\end{aligned}
$$

et $\quad(\mathrm{I}-\theta \mathrm{P})^{-\mathbf{1}} \mathrm{V}$ est le vecteur $\Gamma$

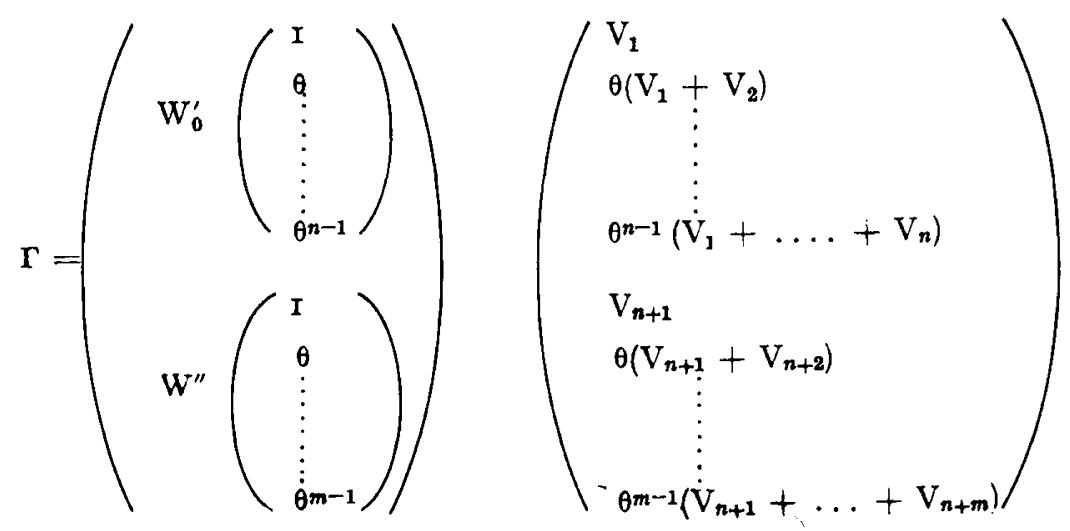


TABLEAU 2

Variables et paramètres caractérisant un schíma de sćlection Characterizing variables and parameters of a selection scheme

\begin{tabular}{|c|c|c|c|c|}
\hline La variable ou le paramètre : & $\pi r$ & $\begin{array}{l}\text { intervient } \\
\mathrm{I}-\theta \mathrm{P}\end{array}$ & Is: & $t$ \\
\hline 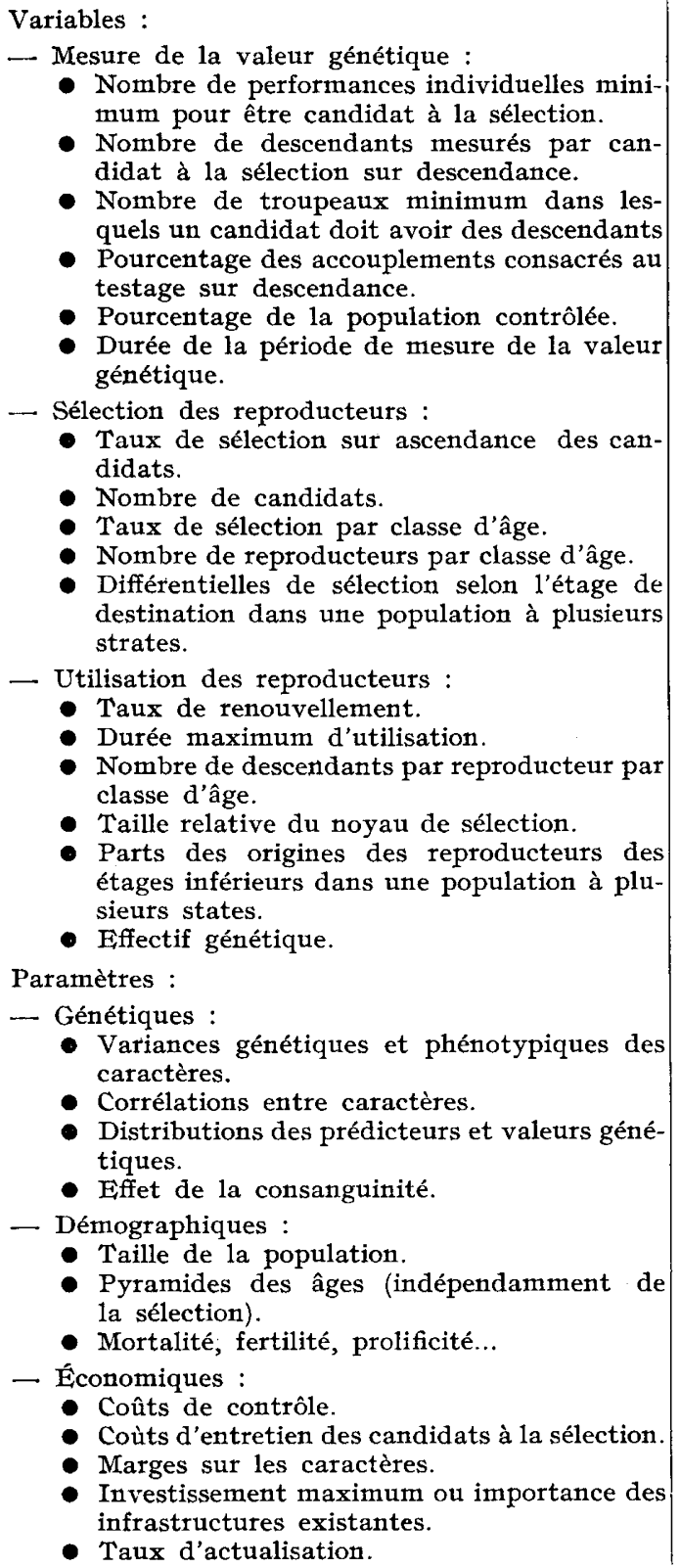 & $\begin{array}{l}\mathrm{X} \\
\mathrm{X} \\
\mathrm{X} \\
\mathrm{X}\end{array}$ & $\begin{array}{l}\mathrm{X} \\
\mathrm{X} \\
\mathrm{X} \\
\mathrm{X}\end{array}$ & $\begin{array}{l}\mathrm{X} \\
\mathrm{X} \\
\mathrm{X} \\
\mathrm{x} \\
\mathrm{X} \\
\mathrm{X}\end{array}$ & $\begin{array}{l}\mathrm{X} \\
\mathrm{X} \\
\\
\mathrm{X} \\
\mathrm{X}\end{array}$ \\
\hline
\end{tabular}

(I) Voir le texte : un schéma de sélection est jugé sur la valeur prise par un critère $\Delta$ fonction des coûts $t$ et des recettes $\mathrm{r}_{2}={ }^{t} r_{\pi r}$. $(\mathrm{I}-\theta \mathrm{P})^{-\mathrm{TV}}$ 
avec

$$
\begin{aligned}
& \mathrm{W}_{0}^{\prime}=\frac{\mathrm{I}}{\delta}\left[\left(\mathrm{I}-\mathrm{D}_{1}\right) \cdot\left({ }^{t r} \mathrm{~A} \cdot \mathrm{V}^{\prime}+{ }^{t r} \mathrm{~B} \cdot \mathrm{V}^{\prime \prime}\right)+\mathrm{B}_{1} \cdot\left({ }^{t r} \mathrm{C} \cdot \mathrm{V}^{\prime}+{ }^{t r} \mathrm{D} \cdot \mathrm{V}^{\prime \prime}\right)\right] \\
& \mathrm{W}_{0}^{\prime \prime}=\frac{\mathrm{I}}{\delta}\left[\mathrm{C}_{1} \cdot\left({ }^{t r} \mathrm{~A} \cdot \mathrm{V}^{\prime}+\operatorname{tr} \mathrm{B} \cdot \mathrm{V}^{\prime \prime}\right)+\left(\mathrm{I}-\mathrm{A}_{1}\right) \cdot\left({ }^{t r} \mathrm{C} \cdot \mathrm{V}^{\prime}+{ }^{t r} \mathrm{D} \cdot \mathrm{V}\right)\right]
\end{aligned}
$$

On a donc une expression, certes compliquée, mais sans inversion de matrice des recettes $\boldsymbol{r}_{2}$ (rappelons que $\pi=(p, q)$ ) :

$$
\mathbf{r}_{2}=\frac{r}{\mathrm{I}-\theta}\left(\mathrm{W}_{0}^{\prime} \sum_{i=1}^{n} \theta^{i} p_{i}+\mathrm{W}_{0}^{\prime \prime} \sum_{j=1}^{m} \theta^{j} q_{j}+\sum_{i=1}^{n} \mathrm{~V}_{i}^{\prime}\left(\sum_{k=i}^{n} \theta^{k} p_{k}\right)+\sum_{j=1}^{m} \mathrm{~V}_{j}^{\prime \prime}\left(\sum_{k=j}^{m} \theta^{k} q_{k}\right)\right)
$$

Une situation très fréquemment étudiée (HInks (I972,) HILL (I97I), LINDHE (I968) ...) est celle où les femelles de renouvellement sont un échantillon aléatoire de l'ensemble des femelles nées, ce que nous traduirons par $\pi=(c, d)$.

Dans ce cas :

$$
\mathrm{r}_{2}=\frac{r}{(\mathrm{I}-\theta) \delta}\left[\mathrm{C}_{1}\left({ }^{t r} \mathrm{~A} \cdot \mathrm{V}^{\prime}+{ }^{t r} \mathrm{~B} \cdot \mathrm{V}^{\prime \prime}\right)+\left(\mathrm{I}-\mathrm{A}_{1}\right) \cdot\left({ }^{t r} \mathrm{C} \cdot \mathrm{V}^{\prime}+{ }^{t r} \mathrm{D} \mathrm{V}^{\prime \prime}\right)\right]
$$

En pratique, l'expression des recettes $\mathbf{r}_{2}$ devra être formulée en fonction des constantes caractéristiques du schéma étudié, et on pourra rechercher l'optimum ou analyser la sensibilité soit en calculant des valeurs points par points, soit en dérivant $\boldsymbol{r}_{2}$ par rapport aux variables ou paramètres.

\section{3. - Populations à plusieurs sous-unités}

Bien qu'aucune solution générale simple existe, la démarche à suivre est toujours la même : partition de $\mathrm{I}-\theta \mathrm{P}$ en blocs et utilisation de la formule directe de $\left(\mathrm{I}-{ }_{\alpha \beta} \mathrm{Q}\right)^{-1}$.

Ainsi, dans le cas des populations de type 2 où :

$$
(\mathrm{I}-\theta \mathrm{P})=\left(\begin{array}{ccc}
\mathrm{I}-\theta_{11} \mathrm{Q} & \mathrm{O} & \mathrm{O} \\
-\theta_{21} \mathrm{Q} & \mathrm{I}-\theta_{22} \mathrm{Q} & \mathrm{O} \\
\mathrm{O} & -{ }_{32} \mathrm{Q} & \mathrm{I}-\theta_{33} \mathrm{Q}
\end{array}\right)
$$

On trouve que :

$$
\Gamma=\left(\begin{array}{l}
{ }_{1} \Gamma \\
{ }_{2} \Gamma \\
{ }_{3} \Gamma
\end{array}\right)=\left(\begin{array}{l}
\left(\mathrm{I}-\theta_{11} \mathrm{Q}\right)^{-1} \cdot{ }_{1} \mathrm{~V} \\
\left(\mathrm{I}-\theta_{22} \mathrm{Q}\right)^{-1} \cdot\left(\theta_{21} \cdot \mathrm{Q} \cdot{ }_{1} \Gamma+{ }_{2} \mathrm{~V}\right) \\
\left(\mathrm{I}-\theta_{33} \mathrm{Q}\right)^{-1} \cdot\left(\theta \cdot{ }_{32} \mathrm{Q} \cdot{ }_{2} \Gamma+{ }_{3} \mathrm{~V}\right)
\end{array}\right)
$$

${ }_{1}^{t r} \pi . r_{1} \Gamma$ étant donné par la formule des recettes dans le cas d'une population de type $I,{ }_{2} \Gamma$ et ${ }_{3} \Gamma$ pouvant être calculés en reprenant la méthode utilisée pour 1'estimation des écarts (II 3) : ${ }_{2} \Gamma={ }_{2} \Gamma-{ }_{1} \Gamma+{ }_{3} \Gamma$ et ${ }_{3} \Gamma={ }_{3} \Gamma-{ }_{2} \Gamma+{ }_{2} \Gamma$. 


\section{IV. - Discussion et conclusion}

Notre objectif dans cet article était de donner quelques outils mathématiques utilisables pour comparer et juger des plans de sélection dans le cas de popularions dont les générations se chevauchent et où existent des phénomènes de migration. Ces outils sont dérivés d'un modèle de prévision et en ont donc les limites qui ont été largement discutées par HILL (I974) et ELSEN et MocQuoT (I974). Rappelons en particulier qu'il est supposé que seules les espérances des distributions des prédicteurs et des valeurs génétiques changent sous l'effet de la sélection donc que celle-ci n'a aucune influence ni sur les variances ni sur les corrélations entre caractères et que les caractéristiques démographiques et économiques sont invariables.

Cette dernière hypothèse a d'ailleurs été critiquée par Hopkins et JAMEs (I979) qui pensent que le plus souvent la matrice de transition P n'est pas constante les premières années de la sélection et soulignent que l'effet d'une seule année de sélection dépend de l'évolution ultérieure de la structure de la population.

Par notre présentation nous avons distingué deux types de prévision : à long et moyen terme. Les premiers résultats correspondent à la démarche classique depuis RENDEL et ROBERTSON (I950) qui consiste à "observer " une population où la sélection est pratiquée depuis longtemps. S'ils sont facilement critiquables, compte tenu des hypothèses inhérantes au modèle, ils ont toutefois la vertu d'être simples et facilement interprétables. En outre, ils quantifient, au moins en partie, la qualité génétique du schéma pratiqué. Les résultats à moyen terme ont par contre une vocation plus économique : on y rend compte de l'échelonnement dans le temps de la réponse à la sélection, phénomène particulièrement important dans les espèces à intervalles de génération long et dans les populations hiérarchisées. D'un point de vue mathématique, les résultats ne sont pas simples mais devraient permettre, en utilisant le calcul automatique, l'étude d'un nombre suffisant de situations pour bien cerner la validité d'une opération de sélection.

Ces deux types de résultats sont d'ailleurs utiles puisque le sélectionneur sera toujours intéressé par le maximum d’informations sur les conséquences de ses décisions.

Reçu pour publication en mai 1980.

\section{Remerciements}

L'auteur remercie le Professeur J.W. JAMES pour ses utiles critiques et suggestions.

\section{Summary}

Genetic progress diffusion in overlapping generations populations: a few properties of a prevision model

The evolution of the mean genetic value of a population under selection, when generations overlap, was first described in 1974 by HILL and by E,LSEN and MOcQUOT using a matrix model whose formulation was close to MARKOV's processes.

In this paper, some interesting properties of this model relative to the case of populations 
divided in sub populations (selection nucleus, multiplication stage and production flocks) are presented in detail.

Concerning long term effects, we have analysed the annual progress to be hoped in each sub population, and the improvement lag between each tier and the nucleus. The case of a population with two stages is particulary detailed and the expressions already obtained by BICHARD in I97I have been generalized.

The short term effects are the most important economically. The proposed model allows a synthetic formulation of the discounted returns of a selection scheme. This formulation is simple but leads to quite long calculations because of a necessary matrix inversion. In order to permit a broader utilisation for rentability studies, a more convenient expression of these incomings is given here.

It is even possible to determine the optimal value of any decision variable by deriving the former expression.

\section{Références bibliographiques}

BICHARD M., r97I. Dissemination of genetic improvement through a livestock industry. Anim. Prod. $13: 401-4$ II.

Brascamp E. W., I973. Model calculations concerning economic optimalization of AI. Breeding with cattle. I. The economic value of genetic improvement in milk yield. Z. Tierziucht. Züchtgsbiol. $90:$ I-I 5 .

Mc Cinntock A. E., Cunningham E. P., I974. Selection in dual purpose cattle population : defining the breeding objective. Anim. Prod. $18: 237-247$.

Cox D. R. and MILLER H. D., 1965. The theory of stochastic processes. Chapman et Hall. London, $398 \mathrm{p}$.

CUNNingham E. P. and JoAn RYAN, 1975. A note on the effect of the discount rate and length of the accounting period on the economic value of genetic improvement in cattle populations. Anim. Prod. 21 : 77-80.

ELSEN J. M., MOCQUO'T J. C., 1974. Recherches pour une rationalisation technique et économique des schémas de sélection des bovins et ovins. Bull. Techn. Dépt. Génét. anim. (17). Inst. Nat. Rech. Agr. France.

ELSEN J. M. et SELLIER P., I979. Étude conjointe de l'intérêt de la sélection sur la prolificité et de l'utilisation d'une lignée mâle spécialisée chez le porc. Ann. Génét. Sél. anim., 10:403-44I.

GaNTMACher F. R., r966. Théorie des matrices. Tome 2. Questions spéciales et applications Dunod, Paris, $268 \mathrm{p}$.

HILI, W. G., I97I. Investement appraisal for national breeding programmes. Anim. Prod. 13, 37-50.

HILL W. G., 1974. Prediction and evaluation of response to selection with overlapping generations. Anim. Prod. 18 : I I 7-I 40.

HINks C. J. M., I972. The effects of continuous sire selection on the structure and age composition of dairy cattle populations. Anim. Prod., 15, 103-1 ro.

Hopkins T. R., JAMES J. W. 1979. Genetic responses in the early years of selection programmes using genetic differences between generations. Anim. Prod., $28: 65-77$.

Hunt M. S., Burnside E. B., Freeman M. G. and Wilton J. W., I974. Genetic gain when sire sampling and proving programs vary in different artificial insemination population sizes. J. dairy Sci, 57, $25^{\mathrm{I}-257}$.

Jackson N., Newton TuRner H., I972. Optimal structure for a cooperative nucleus breeding system. Proc. Aust. Soc. Anim. Prod., $9: 55-69$.

LINDHE B. I968. Model simulation of AI Breeding within a dual purpose breed of cattle. Acta. Agr. Scand. 18, 33-41.

Poutous M. et VISSAC B., r962. Recherche théorique des conditions de rentabilité maximum de l'épreuve de descendance des taureaux d'insémination artificielle. Ann. Zootechn. 11, 233-256.

RENDEI J. M., ROBERTSON A., 1950. Estimation of genetic gain in milk yield by selection in a closed herd of dairy cattle. $J$. Genct., $50: \mathbf{I}-8$.

SEARLE S. R., I962. Bull sampling programs in the artificial breeding of dairy cattle. Proc. New Zeland Soc. Anim. Prod. 22, 54-66.

Surth C., r96o. Efficiency of animal testing schemes. Biometrics 16, 408-4I5. 


\section{Annexe 1. - Expression de la matrice $(I-\alpha \beta Q)^{-1}$}

a) Pour alléger la démonstration nous noterons ici :

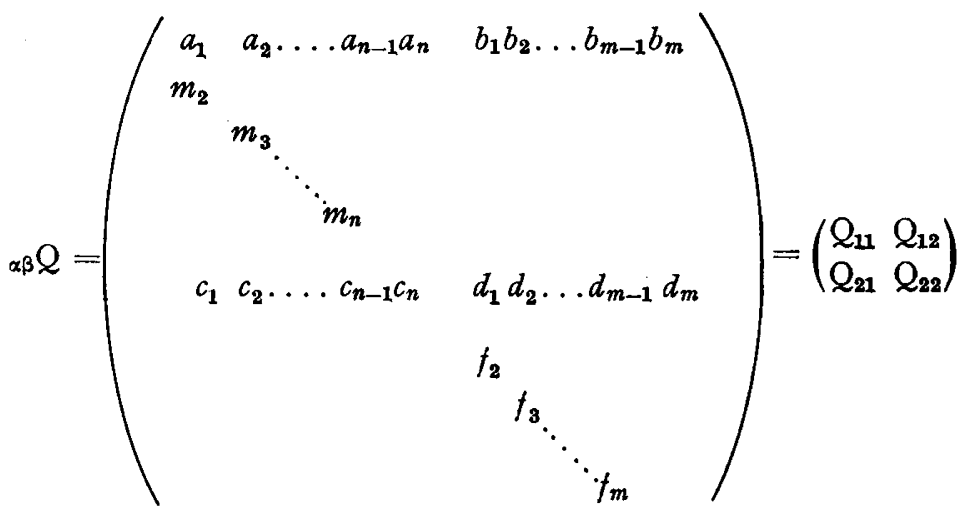

$Q_{11}, Q_{12}, Q_{21}$ et $Q_{22}$ étant les quatre sous-matrices de dimension $n^{2}, n x m$, $m x n$ et $m^{2}$.

Soit par ailleurs $M$ la matrice : $M=\left(\begin{array}{ll}O & Q_{12} \\ Q_{21} & O\end{array}\right)$ et $\mathrm{J}$ la matrice : $\left(\mathrm{I}-{ }_{\alpha \beta} \mathrm{Q}+\mathrm{M}\right)^{-1}$

$$
J=\left(\begin{array}{cc}
I-Q_{11} & O \\
O & I-Q_{22}
\end{array}\right)^{-1}=\left(\begin{array}{cc}
\left(I-Q_{11}\right)^{-1} & O \\
O & \left(I-Q_{22}\right)^{-1}
\end{array}\right)=\left(\begin{array}{ll}
J_{1} & O \\
O & J_{2}
\end{array}\right)
$$

On peut écrire $\left(\mathrm{I}-{ }_{\alpha \beta} \mathrm{Q}\right)^{-1}=\left(\mathrm{J}^{-1}-\mathrm{M}\right)^{-1}=\mathrm{J}\left(\mathrm{I}+\mathrm{MJ}+(\mathrm{MJ})_{2}+\ldots\right)$

En notant : $R_{1}=Q_{12} \cdot J_{2} \cdot Q_{21} \cdot J_{1}$

$$
\text { et : } R_{2}=Q_{21} \cdot J_{1} \cdot Q_{12} \cdot J_{2}
$$

On trouve que $(\mathrm{MJ})^{2}=\left(\begin{array}{cc}\mathrm{R}_{1} & \mathrm{O} \\ \mathrm{O} & \mathrm{R}_{2}\end{array}\right)$ et $(\mathrm{MJ})^{2 k}=\left(\begin{array}{cc}\mathrm{R}_{1}^{k} & \mathrm{O} \\ \mathrm{O} & \mathrm{R}_{2}^{k}\end{array}\right)$

Donc : $\quad\left(\mathrm{I}-\alpha_{\beta} \mathrm{Q}\right)^{-1}=\mathrm{J} \cdot\left(\begin{array}{cc}\left(\mathrm{I}-\mathrm{R}_{1}\right)^{-1} & \mathrm{O} \\ \mathrm{O} & \left(\mathrm{I}-\mathrm{R}_{2}\right)^{-1}\end{array}\right) \cdot\left(\begin{array}{cc}\mathrm{I} & \mathrm{Q}_{12} \mathrm{~J}_{2} \\ \mathrm{Q}_{21} \mathrm{~J}_{1} & \mathrm{I}\end{array}\right)$

b) Il faut donc dans un premier temps trouver les expressions de $\mathrm{J}_{1}$ et $\mathrm{J}_{2}$. Soit alors :

$$
\begin{aligned}
& \mathrm{M}_{11}=\left(\begin{array}{cccc}
a_{1} & a_{2} & \ldots & a_{n} \\
\mathrm{O} & \mathrm{O} & \ldots & \mathrm{o} \\
\vdots & \vdots & & \vdots \\
\mathrm{O} & \mathrm{O} & \ldots & .
\end{array}\right) \\
& \text { et } \quad J_{11}=\left(I-Q_{11}+M_{11}\right)^{-1} \\
& \mathrm{~J}_{1}=\left(\mathrm{I}-\mathrm{Q}_{11}+\mathrm{M}_{11}-\mathrm{M}_{11}\right)^{-1}=\mathrm{J}_{11}\left(\mathrm{I}+\mathrm{M}_{11} \mathrm{~J}_{11}+\left(\mathrm{M}_{11} \mathrm{~J}_{11}\right)^{2}+\ldots\right)
\end{aligned}
$$



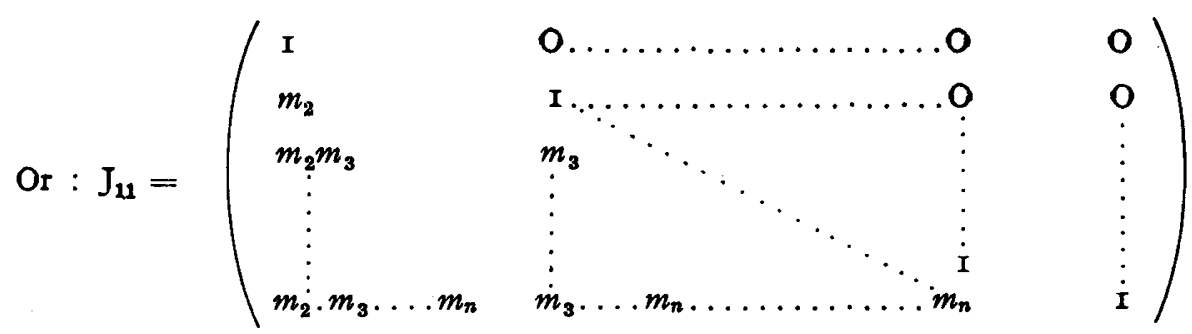

et, en notant :

$$
\mathrm{A}_{i}=\left(a_{i}\right)+\left(a_{i+1} m_{i+1}\right)+\left(a_{i+2} m_{i+1} m_{i+2}\right)+\ldots+\left(a_{n} m_{i+1} \ldots m_{n}\right)
$$

$$
\mathrm{M}_{11} \mathrm{~J}_{11}=\left(\begin{array}{ccc}
\mathrm{A}_{1} & \mathrm{~A}_{2} \ldots \ldots \ldots . \mathrm{A} \\
\mathrm{O} & 0 \ldots \ldots \ldots . \\
\vdots & \vdots & \vdots \\
\vdots & \vdots & \vdots \\
\mathrm{O} & 0 . \ldots \ldots
\end{array}\right)
$$

donc : $\quad \mathrm{M}_{11} \mathrm{~J}_{11}^{k}=\left(\mathrm{A}_{1}\right)^{k} \mathrm{M}_{11} \mathrm{~J}_{11}$

$$
\text { et } \begin{aligned}
\mathrm{J}_{1} & =\mathrm{J}_{11}+\frac{\mathrm{J}_{11} \mathrm{M}_{11} \mathrm{~J}_{11}}{\mathrm{I}-\mathrm{A}_{1}} \\
& =\frac{\mathrm{I}}{\mathrm{I}-\mathrm{A}_{1}}\left[\begin{array}{ccc}
\mathrm{I} & \mathrm{A}_{1} & \ldots \ldots \mathrm{A}_{n} \\
m_{2} & \mathrm{I}-\mathrm{A}_{1}+m_{2} \mathrm{~A}_{2} & \ldots \ldots \ldots m_{2} \mathrm{~A}_{n} \\
m_{2} m_{3} & m_{3}\left(\mathrm{I}-\mathrm{A}_{1}+m_{2} \mathrm{~A}_{2}\right) \ldots \ldots \ldots m_{2} m_{3} \mathrm{~A}_{n} \\
\vdots & \vdots & \vdots \\
\vdots & \vdots & \vdots \\
m_{2} m_{3} \ldots m_{n} & m_{3} \ldots m_{n}\left(\mathrm{I}-\mathrm{A}_{1}+m_{2} \mathrm{~A}_{2}\right) \ldots \mathrm{I}-\mathrm{A}_{1}+m_{2} \ldots m_{n} \mathrm{~A}_{n}
\end{array}\right]
\end{aligned}
$$

De même $\mathrm{J}_{2}=\mathrm{J}_{22}+\frac{\mathrm{J}_{22} \cdot \mathrm{M}_{22} \cdot \mathrm{J}_{22}}{\mathrm{I}-\mathrm{A}_{i}}, \mathrm{D}_{i}, \mathrm{~B}_{i}$ et $\mathrm{C}_{i}$ étant définis comme $\mathrm{D}_{1}$.

c) Connaissant $J_{1}$ et $J_{2}$, on peut en déduire $Q_{12} J_{2}$ et $Q_{21} J_{1}$

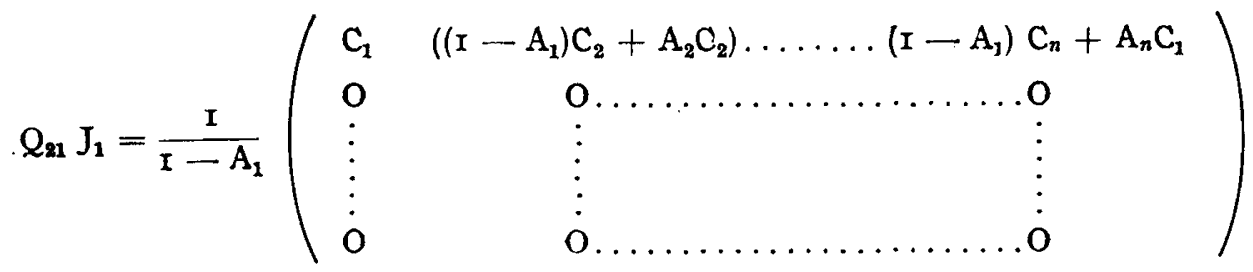

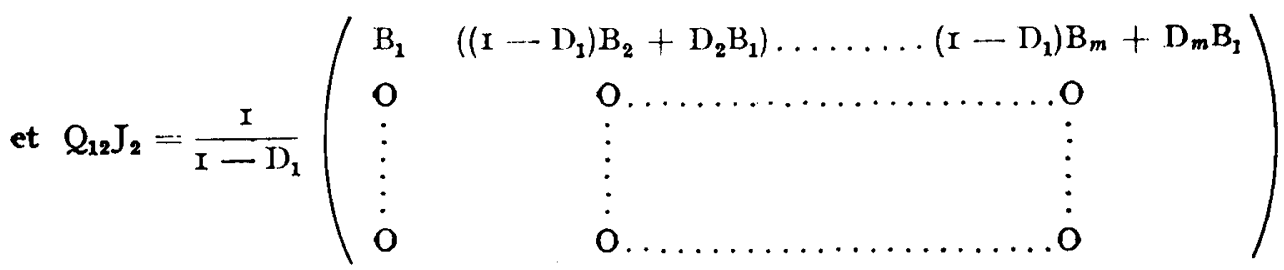


d) On sait alors écrire $R_{1}$ et $R_{2}$

Si en effet, $\psi_{12}$ et $\psi_{21}$ sont les matrices carrées

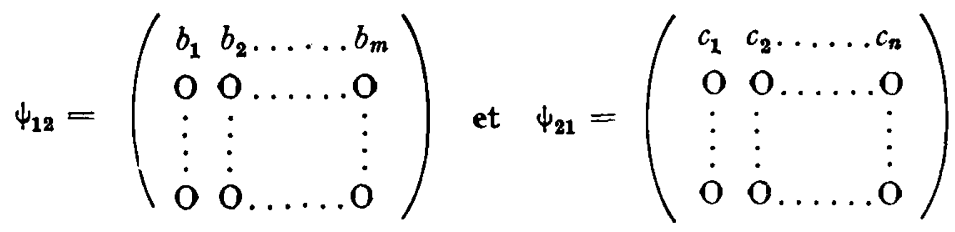

$$
\begin{aligned}
& \mathrm{R}_{1}=\frac{\mathrm{B}_{1}}{\mathrm{I}-\mathrm{D}_{1}} \psi_{21} \mathrm{~J}_{1} \quad \text { et } \quad \mathrm{R}_{2}=\frac{\mathrm{C}_{1}}{\mathrm{I}-\mathrm{A}_{1}} \psi_{12} \mathrm{~J}_{2}
\end{aligned}
$$

e) Alors $\quad \mathrm{R}_{1}^{k}=\left[\frac{\mathrm{B}_{1} \mathrm{C}_{1}}{\left(\mathrm{I}-\mathrm{D}_{1}\right)\left(\mathrm{I}-\mathrm{A}_{1}\right)}\right]^{k} \mathrm{R}_{1} \quad$ et $\quad \mathrm{R}_{2}^{k}=\left[\frac{\mathrm{B}_{1} \mathrm{C}_{1}}{\left(\mathrm{I}-\mathrm{D}_{1}\right)\left(\mathrm{I}-\mathrm{A}_{1}\right)}\right]^{k} \mathrm{R}_{2}$

et $\quad\left(I-R_{1}\right)^{-1}=I+\frac{R_{1}\left(I-C_{1}\right)\left(I-A_{1}\right)}{\delta}=I+\frac{B_{1}\left(I-A_{1}\right)}{\delta} \psi_{21} J_{1}$

et $\quad\left(\mathrm{I}-\mathrm{R}_{2}\right)^{-1}=\mathrm{I}+\frac{\mathrm{C}_{1}\left(\mathrm{I}-\mathrm{D}_{1}\right)}{\delta} \psi_{12} \mathrm{~J}_{2}$

où $\quad \delta=\left(\mathrm{r}-\mathrm{A}_{1}\right)\left(\mathrm{I}-\mathrm{D}_{1}\right)-\mathrm{B}_{1} \mathrm{C}_{1}$

f) On peut maintenant donner une expression de $\left(I-{ }_{\alpha \beta} Q\right)^{-1}$

$\left(\mathrm{I}-{ }_{\alpha \beta} \mathrm{Q}\right)^{-1}=\mathrm{J}+\mathrm{J} \Pi \mathrm{J}$

avec $\quad J=\left(\begin{array}{ll}J_{1} & O \\ O & J_{2}\end{array}\right)$ et $\Pi=\frac{I}{\delta}\left(\begin{array}{ll}\mathrm{B}_{1}\left(\mathrm{I}-\mathrm{A}_{1}\right) \psi_{21} & \left(\mathrm{I}-\mathrm{A}_{1}\right)\left(\mathrm{I}-\mathrm{D}_{1}\right) \mathrm{P}_{12} \\ \left(\mathrm{I}-\mathrm{A}_{1}\right)\left(\mathrm{I}-\mathrm{D}_{1}\right) \mathrm{P}_{21} & \mathrm{C}_{1}\left(\mathrm{I}-\mathrm{D}_{1}\right) \psi_{12}\end{array}\right)$

Annexe 2. - Valeurs propres de la matrice $\alpha<\mathbf{Q}(\alpha \alpha)$

I'essentiel des résultats de cette annexe est dû à $B$. Goffinetr (I).

- Par définition, les valeurs propres de la matrice ${ }_{\alpha \alpha} Q$ annulent le déterminant de I. $\lambda-{ }_{\alpha \alpha} Q=D(\lambda)$.

On trouve que :

$$
\begin{aligned}
& \mathrm{D}(\lambda)=\lambda^{n+m}-\sum_{i=1}^{n} \lambda^{n+m-i} a_{i}-\sum_{j=1}^{m} \lambda^{n+m-j} d_{j} \\
& +\left(\sum_{i=1}^{n} \lambda^{n-i} a_{i}\right)\left(\sum_{j=1}^{m} \lambda^{m-j} d_{j}\right)-\left(\sum_{i=1}^{n} \lambda^{n-i} c_{i}\right)\left(\sum_{j=1}^{m} \lambda^{m-j} b_{j}\right)
\end{aligned}
$$

(I) Biométrie INRA, Toulouse. 
En particulier $\mathrm{D}(\mathrm{I})=\mathrm{O}$ pour toutes les matrices ${ }_{\alpha \alpha} \mathrm{Q}$.

I, a matrice $\alpha \alpha Q$ est primitive si elle n'a qu'une valeur propre de module $I$, soit le réel I. Dans le cas contraire, si $r$ est l'indice imprimitivité, les $r$ valeurs propres de modules I sont :

$$
\lambda_{k, r}=\cos \frac{2 \pi k}{r} \mathbf{i}+\sin \frac{2 \pi k}{r} \text { pour } k=0 \text { à } r-\mathbf{I}(\mathbf{I}=\sqrt{-\mathbf{I}}) .
$$

Pour savoir si $\alpha \alpha Q$ est primitive, il faut donc calculer les valeurs du polynome $\mathrm{D}(\lambda)$ pour $\lambda=\lambda_{1, r}, r=2$ à $n+m$.

- On peut donner une condition suffisante pour que $\alpha_{\alpha \alpha} Q$ soit primitive. Soit ${ }^{t r}\left(r_{1} \ldots r_{n+m}\right)$ un vecteur propre colonne de $\alpha \alpha Q$, correspondant à la valeur propre $\lambda$.

Par définition : $\quad \sum_{i=1}^{n} a_{j} . r_{i}+\sum_{j=1}^{m} b_{j} r_{n+j}=\lambda . r_{1}$

$$
\begin{aligned}
& r_{1}=\lambda r_{2} \\
& \vdots \\
& \sum_{i=1}^{n} c_{i} r_{i}+\sum_{j=1}^{m} d_{j} r_{m+j}=\lambda r_{n+1} \\
& r_{n+1}=\lambda r_{n+2}
\end{aligned}
$$

Et on a le système (I) valable pour toute valeur propre :

$$
\left\{\begin{array}{l}
r_{n} \sum_{i=1}^{n} a_{i} \lambda^{n-i}+r_{n+m} \sum_{j=1}^{m} b_{j} \lambda^{m-j}=\lambda^{n} r_{n} \\
r_{n} \sum_{i=1}^{n} c_{i} \lambda^{n-i}+r_{n+m} \sum_{j=1}^{m} d_{j} \lambda^{m-j}=\lambda^{m} r_{n+m}
\end{array}\right.
$$

S'il existe une valeur propre de module (I), autre que I, on a :

(2)

$$
\left\{\begin{array}{l}
\left|r_{n}\right| \leqslant\left|r_{n}\right| \cdot\left|\sum_{i=1}^{n} a i \lambda^{n-i}\right|+\left|r_{n+m}\right| \cdot\left|\sum_{j=1}^{m} b_{j} \lambda^{m-j}\right| \\
\left|r_{n+m}\right| \leqslant\left|r_{n}\right| \cdot\left|\sum_{i=1}^{n} c_{i} \lambda^{n-i}\right|+\left|r_{n+m}\right| \cdot\left|\sum_{j=1}^{m} d_{j} \lambda^{m-j}\right|
\end{array}\right.
$$

Soit, puisque (3) $\left|\sum_{i=1}^{n} a_{i} \lambda^{n-i}\right| \leqslant \sum_{i=1}^{n}\left|a_{i} \lambda^{n-i}\right|=\frac{1}{2}, \ldots$

$$
\text { (4) }\left\{\begin{array}{l}
\left|r_{n}\right| \leqslant \frac{I}{2}\left(\left|r_{n}\right|+\left|r_{n+m}\right|\right) \\
\left|r_{n+m}\right| \leqslant \frac{I}{2}\left(\left|r_{n}\right|+r_{n+m} \mid\right)
\end{array}\right.
$$

qui implique : $\left|r_{n}\right|=\left|r_{n+m}\right|$ 
Alors, de (2) on obtient :

(5)

$$
\left\{\begin{array}{l}
\left|\sum_{i=1}^{n} a_{i} \lambda^{n-i}\right|+\left|\sum_{j=1}^{m} b_{j} \lambda^{m-j}\right| \geqslant \mathrm{I} \\
\left|\sum_{i=1}^{n} c_{i} \lambda^{n-i}\right|+\left|\sum_{j=1}^{m} d_{j} \lambda^{m-j}\right| \geqslant \mathrm{I}
\end{array}\right.
$$

et (3) et (5) permettent d'écrire que :

(6)

$$
\left\{\begin{array}{l}
\left|\sum_{i=1}^{n} a_{i} \lambda^{n-i}\right|=\sum_{i=1}^{n}\left|a_{i} \lambda^{n-i}\right|=\frac{\mathrm{I}}{2} \\
\left|\sum_{j=1}^{m} b_{j} \lambda^{m-j}\right|=\sum_{j=1}^{m}\left|b_{j} \lambda^{m-j}\right|=\frac{\mathrm{I}}{2} \\
\left|\sum_{i=1}^{n} c_{i} \lambda^{n-i}\right|=\sum_{i=1}^{n}\left|c_{i} \lambda^{n-i}\right|=\frac{\mathrm{I}}{2} \\
\left|\sum_{j=1}^{m} d_{j} \lambda^{m-j}\right|=\sum_{i=1}^{m}\left|d_{j} \lambda^{n-j}\right|=\frac{\mathrm{I}}{2}
\end{array}\right.
$$

Pour que (6) soit vraie, il faut que, dans le plan complexe, tous les vecteurs $a_{i} \lambda^{n-i} \ldots$ soient dans la même direction, ce qui est réalisé, si $r$ est l'indice d'imprimitivité, si et seulement si il y a

$r$ - I $a_{i}$ nuls entre tout $a_{i}$ non nul

$r-$ I $b_{i}$ nuls entre tout $b_{j}$ non nul.

Soit en effet $a_{\mu}$ le premier $a_{i}$ non nul en partant de $a_{1}$. A $a_{\mu}$ correspondent les vecteurs :

$$
a_{\mu} \lambda_{k, r}^{n-\mu}=a_{\mu}\left(\cos \frac{2 \pi k(n-\mu)}{r}+\mathbf{i} \sin \frac{2 \pi k(n-\mu)}{r}\right) \text { pour } k=0, r-\mathbf{I}
$$

et seuls les vecteurs $a_{r+\mu} \lambda_{k, r}^{n-(r+\mu)} a_{2 r+\mu} \lambda_{k, r}^{n-(2 r+\mu)}$

sont dans la même direction que $a_{\mu} \lambda_{k, r}^{n-k}$

Une condition suffisante pour que $\alpha_{\alpha} \mathcal{Q}$ soit primitive est donc que les vecteurs $a, b, c$ et $d$ ne soient pas formés de termes non nuls séparés par un nombre constant de termes nuls.

- Inversement on peut également donner une condition suffisante pour que ${ }_{\alpha x} Q$ soit imprimitive - donc une condition nécessaire pour que $\alpha_{\alpha} Q$ soit primitive.

En effet, si $\alpha \alpha Q$ est telle que seuls les éléments $a_{r}, a_{2 r}, b_{r}, b_{2 r}, c_{r}, c_{2 r}$ et $d_{r}$, $d_{2 r}$ sont non nuls, alors le vecteur colonne

$$
\wedge=\operatorname{tr}\left(\alpha, \lambda_{k, r} \alpha, \lambda_{k, r}^{2} \alpha, \ldots, \quad \lambda_{k, r}^{n-1} \alpha, \alpha, \lambda_{k, r} \alpha, \ldots, \quad \lambda_{k, r}^{m-1} \alpha\right)
$$

avec $k=\mathrm{o}, \mathrm{I} \ldots$ ou $r$ - I, est vecteur propre de ${ }_{\alpha \alpha} \mathrm{Q}$ avec la valeur propre $\lambda_{k, r}^{r-1}$

On remarquera que cette structure de matrice correspond à une situation. où la population peut être séparée en $r$ sous-populations totalement indépenđantes qui s'autorenouvellent sucessivement dans le temps selon un cycle de $r$ périodes élémentaires : les animaux nés à la date $t$ se reproduiront aux dates $t+r, t+2 r$, $t+3^{r}$ cependant que leurs descendants le feront en $t+2 r, t+3^{r} \ldots$ 
- Dans les cas particuliers où la matrice ${ }_{\alpha x} Q$ est imprimitive de période $r$, on notera avec GANTMACHER (I966) que la suite des ${ }_{\alpha \alpha} Q^{t}$ se divise en $r$ suites de limite

$$
{ }_{\alpha \alpha} \mathrm{R}_{k}={ }_{\alpha \alpha} \mathrm{Q}_{k}\left({ }_{\alpha \alpha} \mathrm{Q}^{r}\right)^{\infty}
$$

et on pourra raisonner sur la matrice limite moyenne

$$
\alpha x \mathrm{R}=\sum_{r=0}^{r-i} \frac{\alpha \alpha \mathrm{R}^{k}}{r}
$$

